\title{
Validation of a mesoscale weather prediction model using subdomain budgets
}

\author{
By RONNY PETRIK ${ }^{1 *}$, MICHAEL BALDAUF ${ }^{2}$, HEINKE SCHLÜNZEN ${ }^{3}$ \\ and A L M U T G A S S M A N N ${ }^{1}, \quad{ }^{1}$ Max Planck Institute for Meteorology, Hamburg, Germany; \\ ${ }^{2}$ German Weather Service, Offenbach, Germany; ${ }^{3}$ Institute for Meteorology, Hamburg, Germany
}

(Manuscript received 29 January 2010; in final form 1 April 2011)

\begin{abstract}
AB STRACT
The monitoring of conservation properties is essential for model development and for the investigation of the hydrological cycle. This is especially relevant for models that do not solve equations in flux form and do not apply a finite volume discretization. The conservation properties of the mesoscale model COSMO are evaluated by using a finite volume diagnostic approach. That is the subdomain budget of energy, water mass and total mass are diagnosed in a control volume that can be placed at each site in the model domain and is independent of the grid size. Thus, this diagnostic method has the major advantage that it can be applied to realistic simulations. The application of the diagnostic method to the COSMO model reveals a good preservation of the water mass, but large errors in energy and total mass conservation. The analysis shows to which extent errors in the treatment of thermodynamical processes, numerical filters and moisture advection schemes contaminate the subdomain budgets. In this paper we will show that the application of a saturation adjustment scheme under a fixed volume condition is required for models, which use the non-hydrostatic equations and height-based coordinates. Also, a further extension of the model physics will be introduced and discussed for a realistic test case.
\end{abstract}

\section{Introduction}

It is not sufficient to evaluate the results obtained by a numerical weather prediction (NWP) model in comparison to in situ and remote sensing data observations. Furthermore it is essential to evaluate the physical adequacy of the model (e.g. the falsification approach by Schlünzen, 1997). We devote this paper to the diagnostics of conservation properties for mesoscale models that use limited domains. State of the art models are very complex, as they are based on the non-hydrostatic compressible equations, different discretization schemes, extensive physical parameterizations and various artificial damping mechanisms. Due to the complexity it has become a difficult task to ensure physical constraints like conservation of total energy, Ertel's potential vorticity and mass. Nevertheless conservation properties are very important and need to be diagnosed. For example, the water mass budget plays an essential role for the evaluation of the hydrological cycle.

In this paper we introduce a diagnostic method that determines the time evolution of mass and energy relative to subdomains using a finite volume approach. That is, we diagnose

\footnotetext{
* Corresponding author.

e-mail: ronny.petrik@zmaw.de

DOI: $10.1111 /$ j.1600-0870.2011.00528.x
}

the quantities that should be conserved with respect to welldefined control volumes (CVs), which are located at some place in the model domain. Here we extend the approach of Doms (2004), who was focusing solely on the moisture mass conservation of the 'Lokalmodell' (today known as COSMO) (Doms and Schaettler, 2002). In contrast to earlier studies by Bryan and Fritsch (2002) and Satoh (2003) we will not investigate the temporal evolution of mass and energy as integrated quantities over the whole model domain, but we use a 'local' perspective on the conservation properties as a benchmark for the physical parameterizations and dynamical cores of non-hydrostatic models. The 'local' perspective provides the opportunity to assess limited area models. These models come up with lateral relaxation and upper damping zones, in which the conservation properties are strongly violated. The motivation for our work arises due to the fact that models like, for example, COSMO, ARPS (Xue et al., 2000) and MM5 (Dudhia, 1993) do not apply a flux form in the model equations, as it is done in the WRF (Skamarock et al., 2005) or METRAS (Schlünzen et al., 1997) model. Therefore, conservation is not supposed to be guaranteed by the formulation.

In this paper we use one-dimensional advective tests to analyse the errors of the diagnostic method that develop due to computations on a discrete grid and flux reconstructions. We generalize the diagnostic method for the non-hydrostatic compressible 
model COSMO, that is, we determine the budget equations of the quantities water mass, total mass and energy. The diagnostic method shows that large conservation errors are produced during the simulation of thermodynamic processes. Therefore, we investigate the magnitude and the source of the errors. We figure out that non-hydrostatic models using a height-based coordinate must use a saturation adjustment scheme under a fixed volume approach to achieve conservation. Moreover, the diagnostic method shows that the standard diabatic equations of the COSMO model are insufficient for the simulation of explicit moist convection.

The paper is structured as follows. In Section 2, a diagnostic method is introduced and it is tested in a simple advection test bed. This method will be generalized to the COSMO model in Section 3. In Section 4 the performance of the diagnostic method, applied to the standard COSMO model, is shown using idealized cases. In Section 5 we present a modified formulation of the thermodynamics that are used in the COSMO model. In Section 6 we discuss idealized and realistic simulations using the model physics introduced in Section 5. Summary and conclusions are given in Section 7.

\section{Description of the diagnostic method}

The diagnostic tool is based on the general equation for a scalar quantity $\psi$ in integral form

$\frac{\partial}{\partial t} \int_{V} \psi \mathrm{d} V+\int_{\partial V} \mathbf{f}_{\psi} \cdot \mathrm{d} \mathbf{S}=\int_{V} q_{\psi} \mathrm{d} V$

This means that a quantity $\psi$ in a volume $V$ can be changed, if a volume source $q_{\psi}$ or a flux $\mathbf{f}_{\psi}$ across the boundaries of the volume $V$ occur. To investigate the impact of the model simulated dynamical or physical processes on the subdomain budgets, the fulfilment of eq. (1) needs to be checked. This can be done by discretizing each term in this equation.

\subsection{Numerical implementation}

The expression (1) can be numerically approximated by (Baldauf, 2008):

$\int_{C V} \psi \mathrm{d} V \simeq \sum_{G P \in \mathrm{CV}} \psi_{i, j, k} D_{i, j, k} \Delta V_{i, j, k}$, where $D_{i, j, k}$ is the Jacobian of the coordinate transformation, and

$\int_{\partial V} \mathbf{f}_{\psi} \cdot \mathrm{d} \mathbf{S} \simeq \sum_{G P \in \partial V} \mathbf{f}_{\psi ; i *, j *, k *} \cdot \Delta \mathbf{S}_{i *, j *, k *}$,

where $i *, j *$ and $k *$ are the positions at the corresponding cell faces. Equation (2) is also used to approximate the term on the right-hand side of eq. (1). All approximations are second-order approximations (Ferziger and Peric, 2002). We calculate the expressions (2) and (3) in a well-defined CV containing the grid points (GP) $i \in\left[i_{\min }, i_{\max }\right], j \in\left[j_{\min }, j_{\max }\right]$ and $k \in\left[k_{\min }, k_{\max }\right]$.

The fluxes $\mathbf{f}_{\psi}$ have to be reconstructed. Various interpolation methods were tested to calculate the fluxes (refer to Table 1). The formulas are given for a one-dimensional flux $F_{i+1 / 2}=$ $(u \psi)_{i+1 / 2}$ with a well-known normal velocity. Beside the upwind (UDS) and the central difference method (CDS) a third-order (QUICK) (e.g. Ferziger and Peric, 2002) and the Lax-Wendroff scheme (LW) (Durran, 1999) are listed.

Due to real atmospheric conditions with sharp gradients the various approximations possess a different quality. Therefore, the behaviour of a budget diagnosis with various flux reconstructions has to be investigated in idealized flow tests. We focus exclusively on determining advective fluxes.

\subsection{Test of the diagnostic tool with exact shifting}

As a first test for the diagnostic tool, Baldauf (2008) proposed to shift initial data functions with a constant velocity $u_{0}$ through a one-dimensional grid domain (ideal shifting algorithm). The shifting process of a triangle $\psi_{\text {tri }}(x)$ and a pulse function $\psi_{\text {pul }}(x)$

$\psi_{\text {tri }}(x)=\operatorname{MAX}(-10|x+0.25|+1,0) \quad x \in[0,1]$,

$\psi_{\text {pul }}(x)=\frac{1}{1+\mathrm{e}^{60 *(|x-0.25|-0.12)}} \quad x \in[0,1]$

is simulated inside a domain with $100 \mathrm{GP}$ in the range $x \in[0,1]$ with different Courant numbers $\mu$. Figure 1 displays exemplarily the shifting of the triangle. The functions in eq. (4) are shifted through a $\mathrm{CV}$, which is located in the middle of the domain. It is bounded by the GP $i_{\min }=50$ and $i_{\max }=65$. The number of shifting steps $N_{T}$ is calculated in such a way that the function can fully pass through the $\mathrm{CV}$. The $\mathrm{CV}$ budget is discretized by

Table 1. Flux reconstruction method and their order of spatial approximation

\begin{tabular}{lcl}
\hline Method & Accuracy & \\
\hline UDS & 1 & $F_{i+1 / 2}^{\text {UDS }}=\frac{u_{i+1 / 2}}{2}\left(\psi_{i}+\psi_{i+1}\right)-\frac{\left|u_{i+1 / 2}\right|}{2}\left(\psi_{i+1}-\psi_{i}\right)$ \\
CDS & 2 & $F_{i+1 / 2}^{\text {UDS }}=\frac{u_{i+1 / 2}}{2}\left(\psi_{i}+\psi_{i+1}\right)$ \\
QUICK & 3 & $F_{i+1 / 2}^{\mathrm{QUICK}}=\frac{u_{i+1 / 2}}{16}\left[9\left(\psi_{i+1}+\psi_{i}\right)-\psi_{i-1}-\psi_{i+2}\right]-\frac{\left|u_{i+1 / 2}\right|}{16}\left[3\left(\psi_{i+1}-\psi_{i}\right)+\psi_{i-1}-\psi_{i+2}\right]$ \\
LW & 2 & $F_{i+1 / 2}^{\mathrm{LW}}=\frac{u_{i+1 / 2}}{2}\left(\psi_{i}+\psi_{i+1}\right)-\frac{u_{i+1 / 2}^{2} \Delta t}{2 \Delta x}\left(\psi_{i+1}-\psi_{i}\right)$ \\
\hline
\end{tabular}




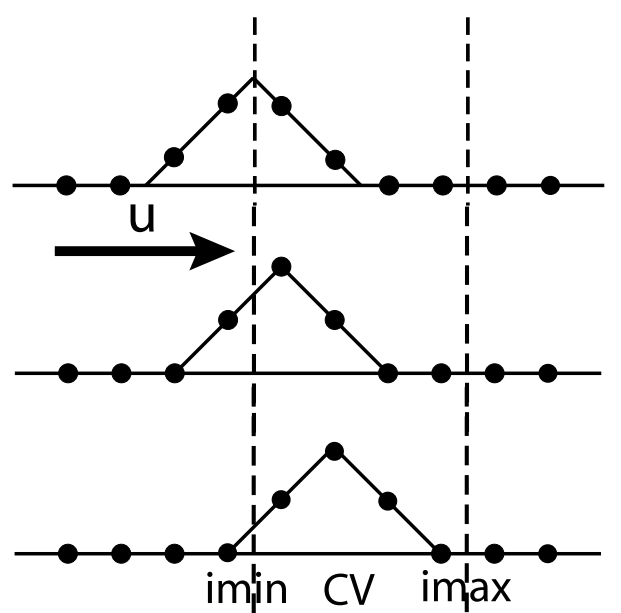

Fig. 1. A triangle shifted one grid point within one time step (Courant number $\mu=1$ ) through a discrete grid. The dashed lines indicate the western and eastern boundary of the control volume.

using eqs. (2) and (3) and the flux interpolation methods listed in Table 1 . The temporal discretization is done by an Euler forward method.

Due to inaccuracies on discrete grids the subdomain budget of the quantity $\Psi^{n}=\sum_{\mathrm{GP} \in \mathrm{CV}} \psi_{i}^{n} \Delta x_{i}$ is not perfectly balanced. To quantify the budget errors the following residual values are defined:

$$
\begin{aligned}
\mathrm{R} & =\frac{\Psi^{n+1}-\Psi^{n}}{\Delta t}+\left(F_{i_{\max }+1 / 2}^{n}-F_{i_{\min }-1 / 2}^{n}\right), \\
\mathrm{IntR} & =\sum_{n=1}^{N_{T}}|\mathrm{R}| \Delta t .
\end{aligned}
$$

Baldauf (2008) showed that even for a perfect shifting process budget errors occur due to flux calculation errors (second term) and volume artefacts (first term on the right-hand side of eq. 5). Thus, the integrated residua IntR listed in Table 2 are mostly nonzero. Although the shifting is perfect, the diagnostic method does not interpret it as perfect. The measurement of the diagnostic tool using the discretizations in eq. (5) are in contradiction to what is going on during the exact shifting process. Only if the Courant number equals one and the flux reconstruction is

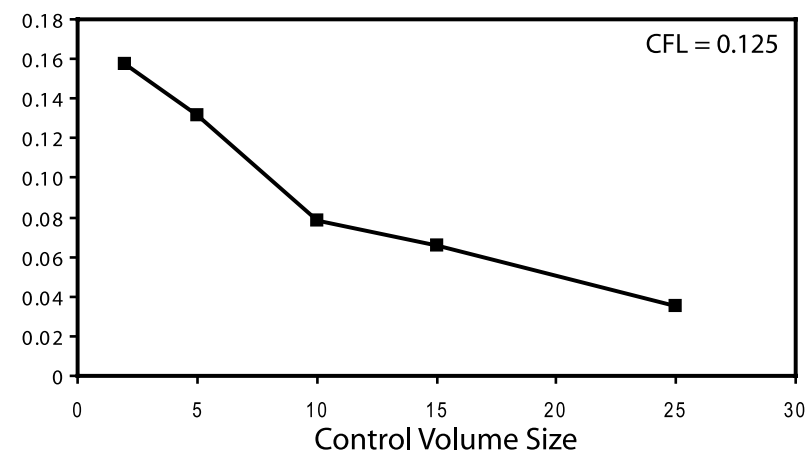

Fig. 2. RE for an exact shifting of the pulse function using various control volume sizes. Average over various budget diagnostics derived from the flux reconstruction methods from Table 1. Values are highly affected from the poor upwind scheme.

done by the UDS or the LW method, the budget error is zero. The shortcomings of the low-order reconstruction schemes are more pronounced, if the shifting process becomes slower or the non-linear pulse function is used instead of the linear triangle function. In this case, the higher order schemes offer lower residua.

To simplify the understanding and comparison of various budget errors, a relative error (RE) normalized with the temporal average of $\Psi^{n}$ is introduced (eq. 6):

$\mathrm{RE}=\frac{\mathrm{IntR}}{{\overline{\Psi^{n}}}^{t}}, \quad{\overline{\Psi^{n}}}^{t}=\frac{1}{N_{T}} \sum_{n=1}^{N_{T}} \Psi^{n}$.

The sensitivity of this error on CV size is shown in Fig. 2 using the pulse function. If the CV size increases the error RE decreases. Enlarging the $\mathrm{CV}$ size increases the volume much more than the total surface area. The determination of the surface flux integral is inaccurate due to flux reconstruction. The volume integral is not affected by flux reconstruction. This means that the impact of inaccurate surface fluxes will reduce, if the $\mathrm{CV}$ gets larger. To obtain reasonable results, the choice of the $\mathrm{CV}$ size must be considered profoundly.

Additionally, the shift test was also extended to 2-D using a slightly stretched grid to mimic the diverging coordinate lines, as they occur in the geographical coordinate system. The integrated

Table 2. IntR for an exact shifting with control volume size of 15 grid points

\begin{tabular}{lccccccc}
\hline & \multicolumn{3}{c}{ Triangle } & & \multicolumn{3}{c}{ Pulse } \\
\cline { 2 - 3 } \cline { 7 - 8 } Method & $\mu=0.125$ & $\mu=0.5$ & $\mu=1$ & & $\mu=0.125$ & $\mu=0.5$ & $\mu=1$ \\
\hline UDS & 0.018 & 0.010 & 0 & & 0.017 & 0.010 & 0 \\
CDS & 0.011 & 0.011 & 0.020 & & 0.003 & 0.010 & 0.020 \\
QUICK & 0.011 & 0.011 & 0.020 & & 0.002 & 0.010 & 0.020 \\
LW & 0.011 & 0.010 & 0 & & 0.002 & 0.001 & 0 \\
\hline
\end{tabular}

Note: Listed for triangle and pulse function with different Courant numbers $\mu$ and flux reconstruction methods. 
residua are qualitatively the same as for the 1-D case, which indicates the ability of the diagnostic method to work in more than one dimension.

\subsection{Test of the diagnostic tool with different numerical advection algorithms}

Due to the fact, that the NWP models apply a numerical integration scheme, the behaviour of the diagnostic tool is applied to different numerical schemes, which compute the advection of the above-mentioned functions (eq. 4 as initial conditions), that is, the analogue to the pure shifting. The time integration is done by a combination of the third-order Runge-Kutta method (Wicker and Skamarock, 2002) used for the temporal discretization and a first-order upstream, a second-order centred and a fifth-order upstream method used for the spatial discretization: RK3UP1, RK3CN2 and RK3UP5. The budget errors (5) are evaluated with the forecasted values $\tilde{\psi}_{i}^{n}$.

It is obvious that the measured numerical transport is not entirely exact (Table 3). Consequently, the shifting with any Courant number and test function reveals errors. The results for the pulse function using the RK3UP5 scheme are nearly the same as in the ideal shifting case. In case of the advection of the triangle, the budget errors are lower than for the ideal shifting (here not shown), because the volume artefacts mentioned in Section 2.2 vanish in the numerical integration and also the measurement by the diagnostic scheme is more in agreement with the numerical advection. Therefore, the subdomain budgets are better when monitoring the RK3UP5 scheme than the pure analytical shifting.

We also test the numerical advection with spatial discretizations lower than fifth order, for example, RK3CN2 and RK3UP1 (Table 3, rows 2 and 3). We analyse how the accuracy of the numerical scheme influences the measurement by the diagnostic method. Indeed, the diagnostic tool is interpreting the simulation of advection using the higher order schemes as much better than using lower order schemes. The lower the spatial discretization order is, the bigger the IntR is. Especially the very dissipative scheme (RK3UP1) is measured with poor conservation proper-

Table 3. IntR for advection of a pulse function using different numerical schemes

\begin{tabular}{lccc}
\hline Numerical scheme & UDS & QUICK & LW \\
\hline RK3UP5, $\mu=0.5$ & 0.001 & 0.001 & 0.001 \\
RK3CN2, $\mu=0.5$ & 0.011 & 0.010 & 0.000 \\
RK3UP1, $\mu=0.5$ & 0.008 & 0.024 & 0.016 \\
RK3UP5WN, $\mu=0.5$ & 0.026 & 0.019 & 0.021 \\
RK2UP5, $\mu=0.9$ & 0.023 & 0.072 & 0.024 \\
\hline
\end{tabular}

Note: Listed for different Courant numbers and flux reconstruction methods. WN indicates white noise. ties concerning the subdomain $\mathrm{CV}$. If the advection is simulated by RK3UP1 and the diagnostic method is using a UDS flux reconstruction for its measurement, then the behaviour of IntR is different due to the coincidence between measuring method and numerical advection scheme.

To simulate numerical artefacts and instabilities, the RK3UP5 solution is disturbed by white noise (RK3UP5WN, amplitude of 0.01) and a Runge-Kutta scheme of second order (RK2UP5) (e.g. Durran, 1999, p. 53-54) is applied above its theoretical stability threshold by using a Courant number of 0.9 . In both cases the IntR indicates detrimental solutions. The white noise and also the unstable scheme RK2UP5 lead to significant higher IntR values compared to the undisturbed solution RK3UP5. Examining the results for the white noise test, the reason for good scores with the 'RK3UP5' solution is not only the perfect symmetry of the pulse function, but also the skills of the numerical advection scheme.

In contrast to the IntR values of the subdomain analysis, the temporal evolution of globally integrated $\psi$ values does not show any problem with the RK3UP5WN and the RK2UP5 integration.

\subsection{Implications of tool tests for its application to complex models}

There is no unique discrete analogon to budget relation (1). Results from pure shifting and advection tests reveal problems in the budget diagnosis due to the discrete grid and the flux reconstruction. To minimize errors originating from the diagnostic scheme itself, the $\mathrm{CV}$ size has to exceed a threshold that needs to be at least $10 \mathrm{GP}$, as depicted in Fig. 2. In addition, we conclude that the higher order reconstruction schemes QUICK and LW (Table 1) are applicable for the following budget investigations with the COSMO model (we use the LW method hereafter). For boundaries of CV close to the surface or the uppermost model level higher order numerical schemes need to be replaced by lower order methods when reconstructing the fluxes.

Our approach of a budget diagnosis with respect to a specific $\mathrm{CV}$ can be applied to those models, which apply a height-based coordinate, even if those models contain complex numerical structures. For instance, if a model is a vertical mass-coordinate model, the $\mathrm{CV}$ is not fixed in space in the vertical, but in mass. The interpretation of the budget may then be a bit different. In order to adapt the diagnostic method to a new model one has to consider two things. First, the diagnostic method has to be adapted to the geometry (Section 3.2.1). Second, the diagnostic method has to be adapted to the equations or to the conservative variables derived from these equations (Sections 3.2.23.2.4).

The diagnostic method is designed for model evaluation. It is voluntarily chosen to be in contrast to the elaborated numerical discretization of the model equations that might only partly be 
budget equations. This allows to detect errors resulting from the model numerics itself.

\section{Adaptation of the diagnostic method to the COSMO model}

\subsection{Relevant model numerics for budget diagnostics}

A short introduction into the formulation and the numerical discretization of the COSMO is given in the Appendix. Here we only address those model features, which will be relevant for the application of the diagnostic method introduced in Section 2.

Due to the fact that the water mass plays a very important role, we will perform sensitivity studies with respect to the moisture transport schemes that are implemented in COSMO (Förstner et al., 2006).

(i) An Eulerian flux-form scheme (BOTT) with a positive definite version of Bott's method (Bott, 1989) and a Strangsplitting technique (chap. 3 Durran, 1999) used to solve the multidimensional advection

(ii) A tri-cubic Semi-Lagrange (SL) advection scheme with full 3-D tri-cubic interpolation within one time step and a multiplicative filling technique (e.g. Staniforth and Cote, 1991)

Moreover, we will study the influence of the numerical filter schemes on the subdomain budgets. The upper model domain is affected by a Rayleigh-damping layer, which extends from the top of the domain $(22 \mathrm{~km})$ down to $13 \mathrm{~km}$ height. A lateral relaxation zone is applied with a width of about $50 \mathrm{~km}$. Furthermore, a fourth-order Laplacian horizontal diffusion is applied to the prognostic variables (standard filter coefficient $v_{l} \approx 1.25 \times 10^{10} \mathrm{~m}^{4} \mathrm{~s}^{-1}$, operationally only applied at the lateral boundaries). The damping of the horizontally propagating sound waves is done using the horizontal divergence damping proposed by Dudhia (1993) (standard filter coefficient $\left.v_{d} \approx 6 \times 10^{4} \mathrm{~m}^{2} \mathrm{~s}^{-1}\right)$.

Taking into account this model configuration, a budget analysis is only valid for a CV far away from the Rayleigh-damping and lateral boundary zones.

\subsection{Examination of the budget equations for the COSMO model}

The model equations of the COSMO are not in flux form. The continuity equation is transformed to a pressure equation. Therefore, the conservation properties are not explicitly ensured and need to be investigated. We apply the diagnostic method of Section 2 to the COSMO model. We focus on the subdomain budgets for total mass, water mass and total energy, because they are conservative variables in the equation system chosen for the COSMO model. That means the volumic sources and sinks vanish, that is, the term on the right-hand side of eq. (1). Only the temporal changes and the boundary fluxes related to the total mass, water mass and total energy have to be determined, that is, the terms on the left-hand side of eq. (1). Therefore, the operators in eqs. (2) and (3) have to be transformed to terms of the coordinate system used in the COSMO. We have to consider the thermodynamic approximations and the macroscopic reference velocity vector $\mathbf{v}$ used in the COSMO model.

3.2.1. Operators. To diagnose the conservation properties following from eq. (1), one needs to define the volume element (eq. 1) of a grid box in a terrain-following system (Baldauf, 2008):

$D_{i, j, k} \Delta V_{i, j, k}=r^{2} \cos \varphi_{j} \sqrt{G}_{i, j, k} \Delta \lambda \Delta \varphi \Delta \zeta$,

The surface integral on the left-hand side of eq. (3) transforms to

$$
\begin{aligned}
\int_{\partial V} & \psi \mathbf{v} \cdot \mathrm{d} \mathbf{S} \\
= & \int_{\partial S^{\varphi, \zeta}} \psi u r \sqrt{G} \mathrm{~d} \varphi \mathrm{d} \zeta+\int_{\partial S^{\lambda, \zeta}} \psi v r \cos \varphi \sqrt{G} \mathrm{~d} \lambda \mathrm{d} \zeta \\
& +\int_{\partial S^{\lambda, \varphi}} r^{2} \cos \varphi\left(\frac{J_{\lambda}}{r \cos \varphi} \psi u+\frac{J_{\varphi}}{r} \psi v-\psi w\right) \mathrm{d} \lambda \mathrm{d} \varphi
\end{aligned}
$$

$\mathrm{d} \mathbf{S}$ is decomposed into $\mathrm{d} \mathbf{S}^{\lambda, \varphi}, \mathrm{d} \mathbf{S}^{\varphi, \zeta}$ and $\mathrm{d} \mathbf{S}^{\lambda, \zeta}$, which are the directed surface elements in the terrain-following coordinate system used in COSMO. Each integral of eq. (8) is discretized by the right-hand side of eq. (3). The values of $\psi$ at the cell face are obtained by reconstruction, which was discussed in Section 2.

3.2.2. Water mass. Wacker and Herbert (2003) and Wacker et al. (2006) studied the budget equations for partial densities of a moist atmosphere, particularly incorporating sedimentation fluxes. Following their approach, the budget of any water component $x$ can be formulated as

$\frac{\partial \rho_{x}}{\partial t}=-\nabla \cdot \rho_{x} \mathbf{v}_{\mathrm{ref}}-\nabla \cdot \mathbf{J}_{\mathrm{ref}, \mathrm{x}}+\sigma_{x}-\nabla \cdot \mathbf{F}_{x}$,

where $\sigma_{x}$ is the internal production rate, for example, cloud water from a condensation process, and $\mathbf{F}_{x}$ is the turbulent mass flux. $\mathbf{J}_{\text {ref }, x}$ is the diffusion mass flux of the component $x$ relative to the macroscopic reference velocity $\mathbf{v}_{\text {ref }}$.

To evaluate the water mass budget in the model runs, the budgets of all partial masses $\rho_{x}$ given in eq. (9) have to be summed up except dry air (abbreviatory $d$ ). Applying the most sophisticated cloud microphysics we consider six classes of water: water vapour $(x=v)$, cloud water $(x=c)$, cloud ice $(x=i)$, rain $(x=r)$, snow $(\mathrm{x}=\mathrm{s})$ and graupel $(\mathrm{x}=\mathrm{g})$. Using the Gaussian theorem the budget for the water mass $M_{\mathrm{W}}$

$M_{\mathrm{W}}=\int_{\mathrm{CV}} \sum_{x \neq d} \rho q_{x} \mathrm{~d} V \equiv \int_{\mathrm{CV}} \rho q_{\mathrm{W}} \mathrm{d} V$ 
in a pre-defined volume $\mathrm{CV}$ is given by

$$
\begin{aligned}
\frac{\partial M_{\mathrm{W}}}{\partial t}= & -\int_{\partial \mathrm{CV}} \rho q_{\mathrm{W}} \mathbf{v} \cdot \mathrm{d} \mathbf{S}_{C V}-\int_{\partial S_{\mathrm{CV}}^{\lambda, \varphi}}\left(F_{v}+F_{c}+F_{i}\right) \mathrm{d} S_{\mathrm{CV}}^{\lambda, \varphi} \\
& +\int_{\partial S_{\mathrm{CV}}^{\lambda, \varphi}} a\left(P_{r}+P_{s}+P_{g}\right) \mathrm{d} S_{\mathrm{CV}}^{\lambda, \varphi}
\end{aligned}
$$

where $S_{\mathrm{CV}}^{\lambda, \varphi}$ denotes the surfaces at the top and the bottom of the CV. The water budget given by eq. (11) is checked by the diagnostic method.

The sub-domain budget (11) is derived as follows:

(i) With respect to the COSMO model, the reference velocity is chosen as a barycentric velocity of total air mixture, that is,

$\mathbf{v}_{\mathrm{ref}} \equiv \mathbf{v}=\sum \frac{\rho_{x}}{\rho} \mathbf{v}_{x}, \quad \rho=\sum \rho_{x}, \quad \rho_{x}=\rho q_{x}$.

(ii) The summation over all source rates $\sigma_{x}$ vanishes to retain the water mass conservation. The dry air source rate is zero.

(iii) We consider the strictly vertical directed mass fluxes of diffusion and turbulence: $\mathbf{J}_{\text {ref }, x}=J_{x} \mathbf{k}$ and $\mathbf{F}_{x}=F_{x} \mathbf{k}$.

(iv) With respect to the precipitating particles, the vertical components of the diffusion fluxes are approximated by the sedimentation fluxes with mean terminal velocities: $J_{x}=-P_{x}, x=$ $r, s, g$. The sedimentation fluxes cause a compensating lift of all non-precipitating classes (the corresponding fluxes $J_{d}, J_{v}, J_{c}$ and $J_{i}$ are defined by Catry et al., 2007). The summation over the diffusion fluxes of the water species gives the third term on the right-hand side of eq. (11). Parameter $a$ is $q_{d} /\left(1-q_{r}-q_{s}-q_{g}\right)$.

(v) The turbulent transport of the precipitating particles is disregarded: $F_{r}=F_{s}=F_{g}=0$.

Taking the impact of water vapour evaporation and precipitation into account, the barycentric velocity is not zero at the surface. Although the Earth surface is impermeable for dry air, the diffusive flux of water vapour $J_{v}$ and the sedimentation flux of the precipitating particles across the Earth surface account for a nonvanishing barycentric velocity at ground (Wacker and Herbert, 2003): $w_{s}=\left(J_{v}-P_{r}-P_{s}-P_{g}\right) / \rho_{d}$. Therefore, the turbulent fluxes and precipitation rates of eq. (11) have to be multiplied by a factor of $\rho / \rho_{d}$, if the lower surface of the $\mathrm{CV}$ is equal to the Earth surface.

If one uses parameterized convection with mass flux schemes, an investigation of the budget given by eq. (11) is somewhat restricted in terms of the location of the $\mathrm{CV}$ in the threedimensional space. In this case only the bulk precipitation is available and an analysis must be carried out for a $\mathrm{CV}$ with a vertical extent from the model surface to the cloud top.

3.2.3. Total mass. The total mass budget can be derived from expression (9) summing up over all components of the air mixture. We use the reference velocity from eq. (12) and apply the necessary mass control conditions $\sum \mathbf{J}_{\mathrm{ref}, x}=0$ and $\sum \mathbf{F}_{x}=0$ (Wacker et al., 2006). Then, the budget for the total mass $M$ is given by

$\frac{\partial}{\partial t} \int_{\mathrm{CV}} \rho \mathrm{d} V \equiv \frac{\partial M}{\partial t}=-\int_{\partial \mathrm{CV}} \rho \mathbf{v} \cdot \mathrm{d} \mathbf{S}_{C V}$.

The total mass budget given by eq. (13) is checked by the diagnostic method.

As already mentioned in Section 3.2.2, the diffusive flux of water vapour and the sedimentation fluxes of the precipitating particles lead to a non-vanishing barycentric vertical velocity at the ground. If we consider the total mass budget in eq. (13) with respect to a $\mathrm{CV}$, where the lower surface is equal to the Earth surface, then the contribution to the mass flux at the Earth surface (index $s$ ) reads explicitly:

$+\left.\frac{\rho_{s}}{\rho_{d, s}} \int_{\partial S_{\mathrm{CV}}^{s}}\left(J_{v}-P_{r}-P_{s}-P_{g}\right)\right|_{s} \mathrm{~d} S_{\mathrm{CV}}^{s}$.

Thus, the atmosphere loses mass, if precipitation is higher than evaporation. Although the COSMO and any other model allow for a diffusive water flux from the soil into the atmosphere, they cannot describe the influence of the vertical boundary velocity $w_{s} \neq 0$ on the prediction of the prognostic variables (e.g. the influence of $w_{s}$ on the surface pressure Wacker et al., 2006). This leads to a lack in the mass conservation.

3.2.4. Energy. The combination of the hydrothermodynamical equations for temperature, pressure, momentum and the budget equations for the mass fractions $q_{x}$ (Steppeler et al., 2003, eqs. 1-5) gives the budget for energy diagnostics (Gassmann and Herzog, 2008):

$$
\begin{aligned}
& \frac{\partial}{\partial t} \int_{\mathrm{CV}} \rho E_{t} \mathrm{~d} V \\
& \quad=-\int_{\partial \mathrm{CV}}\left(\rho E_{t} \mathbf{v}+\overline{\rho \mathbf{v}^{\prime \prime} \mathbf{v}^{\prime \prime}} \cdot \mathbf{v}-p \mathbf{v}+\mathbf{R}+\overline{\rho e^{\prime \prime} \mathbf{v}^{\prime \prime}}\right) \cdot \mathrm{d} \mathbf{S}_{\mathrm{CV}}
\end{aligned}
$$

The energy budget given by eq. (15) is checked by the diagnostic method.

The total mass-normalized energy $E_{t}=K_{m}+\Phi+e$ is the sum of mean kinetic energy $K_{m}=\left(u^{2}+v^{2}+w^{2}\right) / 2$, potential energy $\Phi=g z$ and internal energy $e$. The mean turbulent kinetic energy (TKE) is not considered for the energy budget given in eq. (15). The TKE is used for the turbulence closure concept in the COSMO to yield turbulent transfer coefficients for momentum and heat. However only the transport of turbulent heat fluxes and turbulent momentum fluxes influence the temporal evolution of total energy.

We evaluate only the vertically directed radiation flux density $\mathbf{R}=R \mathbf{k}$. It describes the energy gain in the daytime and the cooling of the atmosphere or the cloud backscattering by longwave radiation. The term $\nabla \cdot p \mathbf{v}$ considers the rate at which work is done by the pressure force (the contraction or expansion of a volume), which changes the internal energy. As already known from mass treatment, surface integrals at the ground must be treated with the barycentric velocity $w_{s}$. Consequently, the surface integral at the Earth surface (index $s$ ) given in 
eq. (15) consists of

$\rho_{s} E_{t, s} w_{s}$ and $p_{s} w_{s}$.

The first term describes, for instance, the input of latent energy to the atmosphere due to strong evaporation fluxes (positive $w_{s}$ ). The second term describes the work done at the Earth surface and used to expand the $\mathrm{CV}$ under the condition that $w_{s} \neq 0$. We name both effects an energy surface flux hereafter.

The internal energy $e$ results from the common relation between pressure $p$ and enthalpy $h$.

$\rho e=\rho h-p, \quad h=\sum h_{i} q_{i}$.

The enthalpy changes during phase transitions, which is reflected by the heat of vapourization $l_{v}$, fusion $l_{f}$ and sublimation $l_{s}$. The expression for $e$ and $h$ are specified following thermodynamical textbooks (e.g. Emanuel, 1994). However, under the condition that different phases of water coexist in the atmosphere, no unique formula for the enthalpy exists. We are following Satoh (2003) to define the origin of the energy $e$.

In the COSMO model a simplified thermodynamic is applied. That is, $l_{v}, l_{f}$ and $l_{s}$ are no longer temperature dependent, rather they are fixed at their values at $0^{\circ} \mathrm{C}$. This leads to an underestimation of the sensible heat storage (Satoh, 2003). As a consequence of Kirchhoff's law of thermodynamics, the heat capacities of vapour, liquid and frozen particles do not contribute to the total $c_{p}$ and $c_{v}\left(c_{p} \simeq c_{p d}\right.$ and $\left.c_{v} \simeq c_{v d}\right)$. This leads to the simplified, but still consistent, expression for the internal energy.

$e=c_{v d} T+l_{v} q_{v}-l_{f} q_{f}, \quad e_{s}=c_{v d} T, \quad e_{l}=l_{v} q_{v}-l_{f} q_{f}$.

$e_{s}$ is the sensible and $e_{l}$ the latent heat part of internal energy. The negative sign in the latent heat part describes that latent energy is stored in the water vapour and released by condensation and freezing.

We have to point out here, that the energy budget diagnostic must be consistent with the thermodynamical assumption in a model. With respect to the COSMO model, the energy budget given by eq. (15) has to be analysed with the internal energy expression (18).

\section{Investigation of subdomain budgets for 3-D idealized cases}

\subsection{Definition of residual budget}

For our following argumentations it is assumed, that the model (index $M$ ) is not perfect and any budget is contaminated by a residuum.

$$
\left.\frac{\partial \psi}{\partial t}\right|_{M}=\left.F_{\psi}\right|_{M}+R_{\psi},
$$

where $F_{\psi}$ denotes fluxes and sources defined by the right-hand side of the eq. (11), (13) and (15). If $R_{\psi}>0$, the model artifi- cially gains mass or energy, whereas $R<0$ means, the model erroneously loses energy or mass.

As discussed in Section 2, the temporal discretization in the budget equations is discretized by an Euler Forward method. All required terms are evaluated in the model at the end of a time step.

\subsection{Experimental setup of the 3-D academic cases}

As a first simple test case a single cell convection was chosen for the analysis of the COSMO model. The pre-convective hydrostatic balanced environment is defined by Weisman and Klemp (1982). In order to overcome the convective inhibition the convection is forced using a warm air bubble. The size and amplitude of the bubble are defined by Weisman and Klemp (1982).

The simulation is performed on a domain of $100 \times 100 \mathrm{GP}$ with a horizontal grid size of nearly $1.9 \mathrm{~km}$. We use 48 model levels, which are vertically stretched to better resolve the boundary layer. For simplification the Coriolis parameter is set to zero. A short overview about the model configuration is given in Table 4.

\subsection{Case WKDRY: dry convection}

We simulate a dry convection. Thus, any phase changes of water vapour are prohibited. In this case $q_{v}$ might be interpreted as a tracer. However it is not a real passive scalar, because it contributes to the total density of the multiphase atmospheric fluid and also influences the buoyancy of the fluid. In contrast to Weisman and Klemp (1982), the initialized warm air bubble has an amplitude of $10 \mathrm{~K}$.

The thermal bubble rises explosively. Within $6 \mathrm{~min}$ its centre reaches $3000 \mathrm{~m}$ height. The maximum vertical velocity is about $10 \mathrm{~m} \mathrm{~s}^{-1}$. The resulting pressure perturbations, in combination with the motions caused by continuity reasons, lead to a rotation in the $y$-z-plane (Fig. 3a).

Table 4. Configurations of the COSMO model for the performed experiments

\begin{tabular}{lccc}
\hline Experiment & WKDRY & WKRAIN & REAL \\
\hline Time step [s] & 20 & 20 & 30 \\
Resolution [km] & 1.9 & 1.9 & 2.8 \\
$\nu_{l}\left[\mathrm{~m}^{4} \mathrm{~s}^{-1}\right]$ & $1.25 \cdot 10^{10}$ & $1.25 \cdot 10^{10}$ & 0 \\
$\nu_{d}\left[\mathrm{~m}^{2} \mathrm{~s}^{-1}\right]$ & $6 \cdot 10^{4}$ & $6 \cdot 10^{4}$ & $6 \cdot 10^{4}$ \\
Moist. advec. & BOTT & BOTT & BOTT + SL \\
Physics & no & cloud micro & $\begin{array}{c}\text { cloud micro + } \\
\text { turbulence + } \\
\text { radiation }\end{array}$ \\
\hline
\end{tabular}

Note: $v_{l}$ denotes the Laplacian filter coefficient and $v_{d}$ the divergence damping coefficient. 
(a)

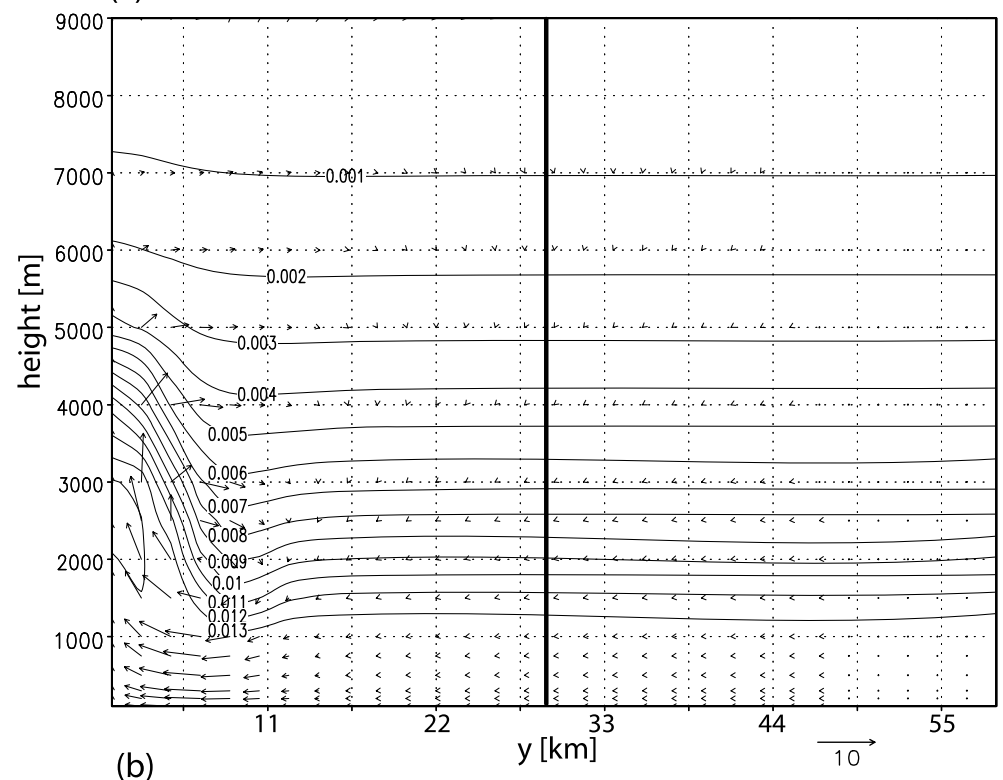

(b)

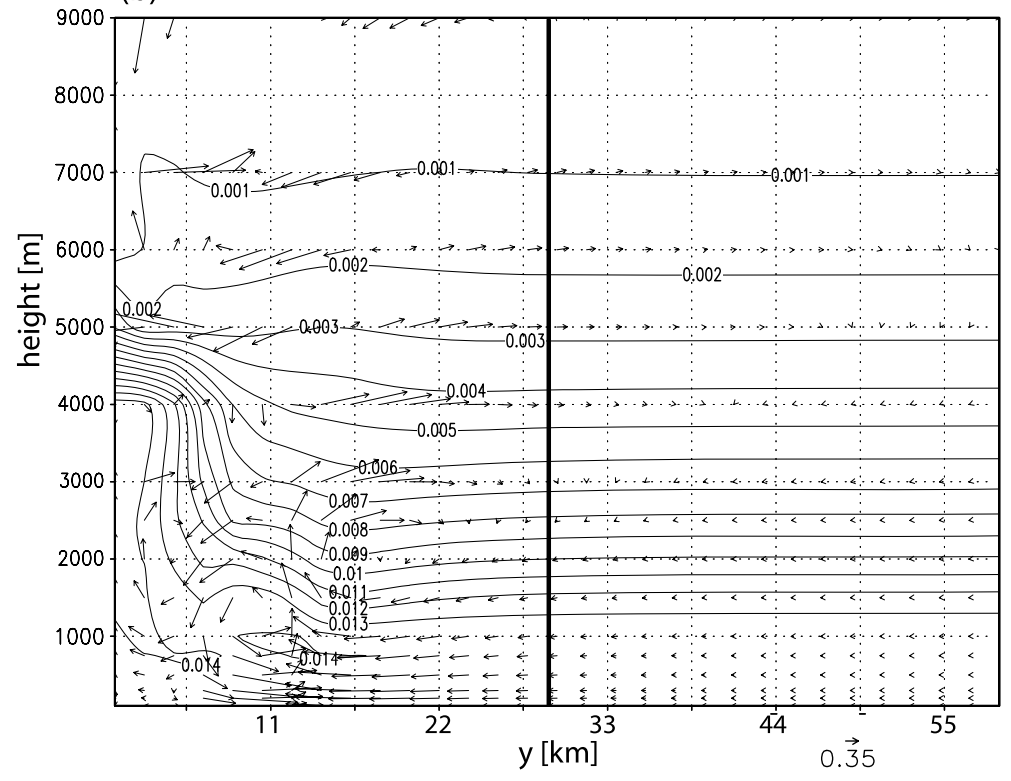

Fig. 3. Case WKDRY. (a) Vertical cross-section at $x=50$ with the centre of the convective cell at the left boundary. Specific water vapour $\left(\mathrm{kg} \mathrm{kg}^{-1}\right)$ (contour) and wind vectors at $t=6$ min. (b) As in (a), but at $t=24 \mathrm{~min}$. The thick line indicates the northern boundary of $\mathrm{CV}$.
Although the rising bubble was initialized with a $10 \mathrm{~K}$ temperature perturbation in its centre, it loses its buoyancy very fast. The resulting descending air cuts the updraft into two pieces, that forms sharp wind fronts in the lower atmosphere. Figure $3 \mathrm{~b}$ illustrates such a front $15 \mathrm{~km}$ to the north and 24 min after initialization. During the first hour the bubble oscillates in a statically stable atmosphere because of the buoyancy force. Later on, this process weakens.

The budget analysis was carried out for a CV with a horizontal extent of $30 \times 30 \mathrm{GP}$ and 31 layers in the vertical from the Earth's surface to nearly $10 \mathrm{~km}$ height. The thermal bubble is located in the centre of the CV. The total mass fluxes through the boundaries of the volume are shown in Fig. 4. All the lateral fluxes are the same due to the missing Coriolis force. The fluxes are significantly influenced by gravity wave generation and show a phase shift between the horizontal and vertical fluxes. An upward mass flux at the top of the $\mathrm{CV}$ is associated with an vertical integrated horizontal convergence and vice versa.

Since the bubble sucks the dense air underneath, that is, a low-level convergence due to its buoyancy at the beginning of the simulation, the total mass is increasing rapidly in the $\mathrm{CV}$. Figure 5a shows the subdomain budget of the total mass. It can be seen that the positive mass change is well explained by the mass fluxes computed at the lateral boundaries. That is the horizontal convergence of mass near the surface is stronger than the loss of mass aloft (Fig. 4). It is important to note, that the 


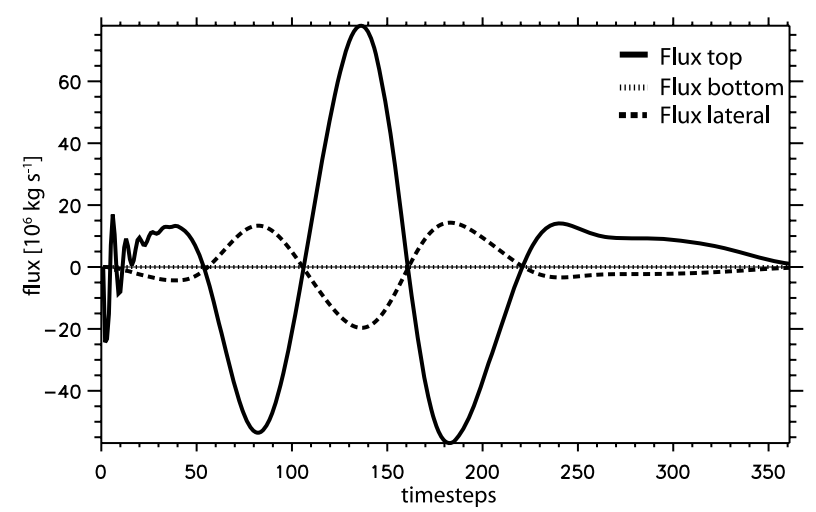

Fig. 4. Case WKDRY $(\Delta t=20 \mathrm{~s})$. Total mass fluxes through the boundaries of the $\mathrm{CV}\left[\mathrm{kg} \mathrm{s}^{-1}\right]$. The term 'Flux lateral' is related to only one single lateral boundary flux (north, south, west and east).

term 'DivFlux_M' in Fig. 5a summarizes the horizontal and vertical part of the mass fluxes, that is, the mass divergence as a three-dimensional quantity.

The spin-up phase is disturbed by vigorous oscillations in the levels near the tropopause $(12 \mathrm{~km}$ ). The RE (eq. 6) in the simulation is about $7 \times 10^{-5}$ and is considered small. The residual curve is smaller than any physical tendency. The remaining error might originate from the inaccuracy of our diagnostic method (see Section 2).

The water mass is well conserved in the chosen CV (Fig. 5b). The ascending air increases the mass of moist air and generates a moisture flux convergence. At the time, when the gravity waves and outflow structures reach the CV boundaries near the surface, the convergence turns into a divergence. The BOTT advection scheme produces oscillations at the beginning. They originate from splitting errors associated with the multidimensionality of the implemented BOTT-advection scheme (for efficiency reasons the splitting steps in the different spatial directions in terms of the Strang-splitting are reduced). If one takes into account the virtual temperature to calculate the CAPE (convective available potential energy Doswell and Rasmussen, 1994), the numerical perturbation in $q_{v}$ means a $2 \mathrm{~J} \mathrm{~kg}^{-1}$ switch in every time step. This is not significant in comparison to the absolute value, but it could be relevant in weather situations with lower CAPE and strong advection processes-for example, fronts in mountainous regions.

\subsection{Case WKRAIN: convection with precipitation}

We push further the idealized tests and perform simulations allowing for precipitation physics. A Warm Rain Kessler scheme is used (Doms et al., 2002, Section 5.4) in order to consider the autoconversion, accretion and evaporation of rain water. The nucleation of cloud water from the vapour phase is done by a standard saturation adjustment scheme. For more details we refer the reader to Doms et al., 2002, (Section 5). The initial atmospheric conditions are slightly modified in comparison to CASE WKDRY. The surface temperature is $T_{s}=297 \mathrm{~K}$. The CAPE is about $1000 \mathrm{~J} \mathrm{~kg}^{-1}$. The warm air bubble has an initial temperature amplitude of $2 \mathrm{~K}$.

The temporal evolution of the simulation at the centre of the convection is shown in Fig. 6. The bubble does not rise as fast as in the WKDRY case during the first minutes, because the temperature excess in the bubble centre is divided by 5 in comparison to the previous run. However, once the condensation starts, the bubble becomes much warmer thanks to the latent heat release. The inflow of moist air is nearly two times stronger than in the WKDRY run. The temperature excess in the centre of the bubble reaches $5 \mathrm{~K}$ and the maximum vertical velocity is $17 \mathrm{~m} \mathrm{~s}^{-1}$ after $45 \mathrm{~min}$. Above the equilibrium level the moist air dries out and is causing an upper tropospheric outflow divergence. When the formation of rain water starts, the liquid water drag increases. This decelerates the flow into the updraft. Later on, the sedimenting rain water reaches the ground. The precipitation intensifies reaching its maximum rate after $60 \mathrm{~min}$. The
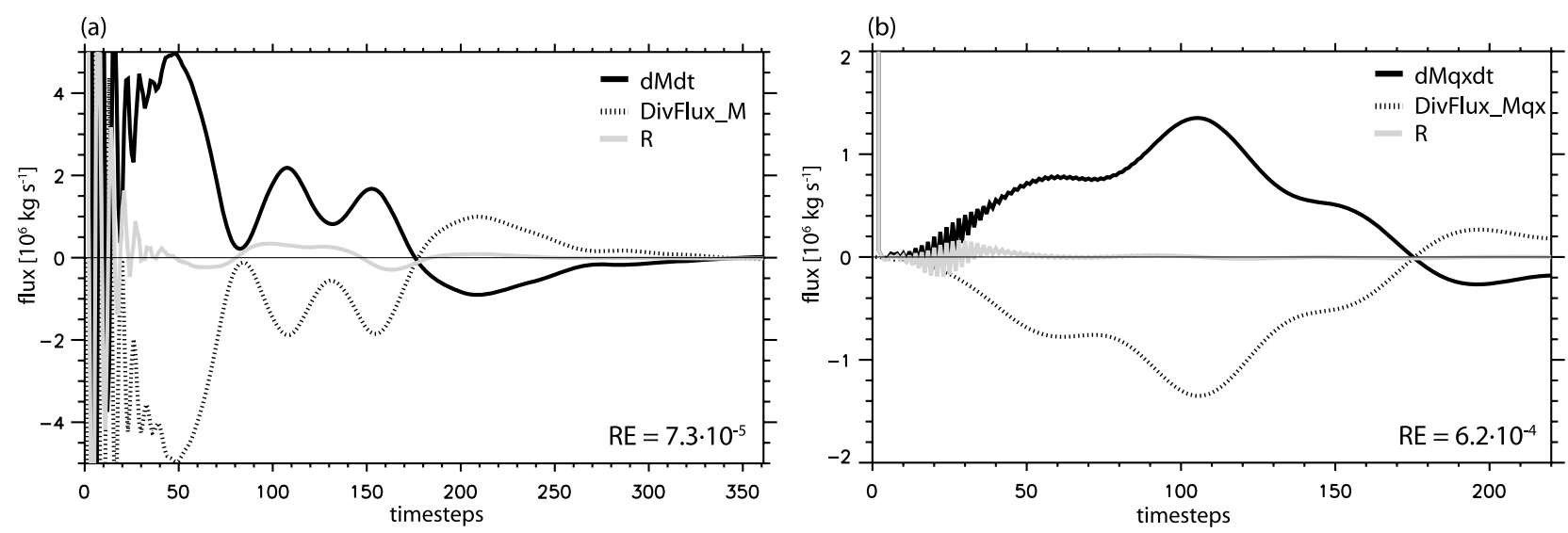

Fig. 5. Case WKDRY ( $\Delta t=20 \mathrm{~s})$. Sub-domain budgets $\left(\mathrm{kg} \mathrm{s}^{-1}\right)$ of the CV. (a) Total mass budget (eq. 13) containing mass change (named 'dMdt'), divergence ('DivFlux_M') and residua ('R'). (b) As in (a), but for the water mass budget (eqs. 10 and 11). 


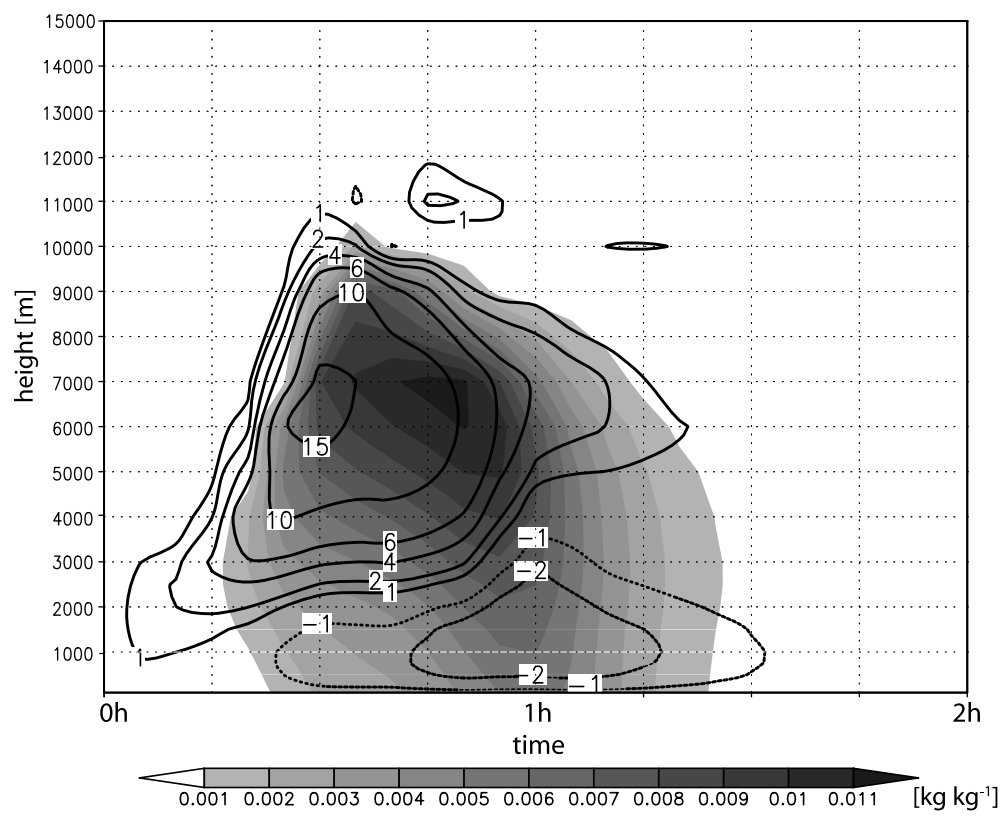

Fig. 6. Case WKRAIN. Time-height cross section of vertical velocity $\left(\mathrm{m} \mathrm{s}^{-1}\right)$ (contour) and rain water content $\left(\mathrm{kg} \mathrm{kg}^{-1}\right)$ (shaded) at the centre of the density current.

developing downdraft cuts off the inflow of moist and dense air and the cell dies.

The total mass budget analysis for the case WKRAIN is presented in Fig. 7a using the same CV as in the case WKDRY. Compared to the WKDRY simulation, the total mass budget clearly shows residua with high negative values. The evolution of the total mass is not explained by the mass fluxes which are computed. One possible explanation is that the total mass, which has been supposed conservative without any volumic sources or sinks (right-hand side term of eq. 1 vanishes), is not conserved by the model. That means 'fictive' and spurious volumic sources or sinks exist inside the $\mathrm{CV}$ due to the discretization or the model approximations. Another explanation is that some 'fictive' inflows or outflows into the $\mathrm{CV}$ are present in the model, which should not exist. There is no divergent flux apparent during the first half hour (the first 90 time steps), although the decrease in total mass will imply it.

The error in total mass has its maximum at about 90 time steps. At that time the highest amount of latent heat (positive diabatic heating) is released inside the CV. Since the cloud formation process is controlled by the standard saturation adjustment scheme, it is supposed to be the main origin for the residual values. We discuss this issue in the next Section.

During the second half of the first hour the residuum curve becomes smaller. Its sign changes after 50 min simulation time (150 time steps). At that time, the evaporation of rain water becomes the dominant physical process and causes a negative diabatic heating.

In order to prove whether the precipitation process also affects the mass and energy conservation, we investigate the subdomain
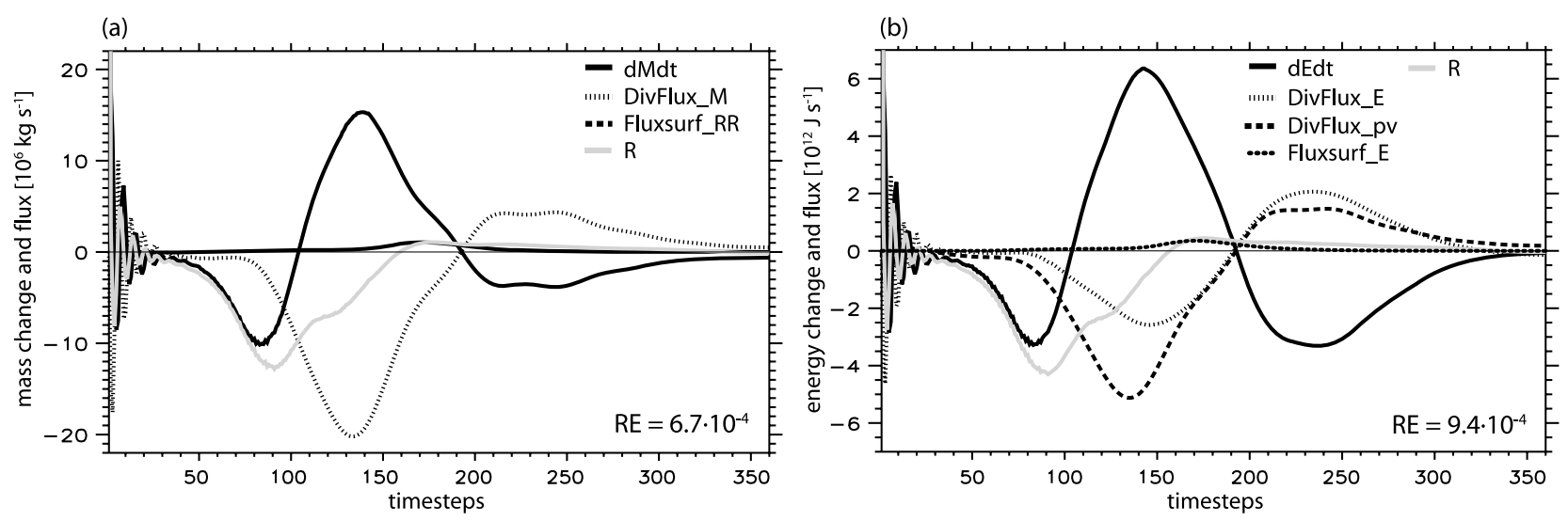

Fig. 7. Case WKRAIN $(\Delta t=20 \mathrm{~s})$. Subdomain budgets of the CV. (a) Total mass budget $\left(\mathrm{kg} \mathrm{s}^{-1}\right)$ involving precipitation flux (named 'Fluxsurf_RR', eq. (14); (b) as in (a), but for the total energy ( $\mathrm{J} \mathrm{s}^{-1}$ ) budget involving the work done by the CV ('DivFlux_pv') and the energy surface flux ('Fluxsurf_E', eqs. 15 and 16). 


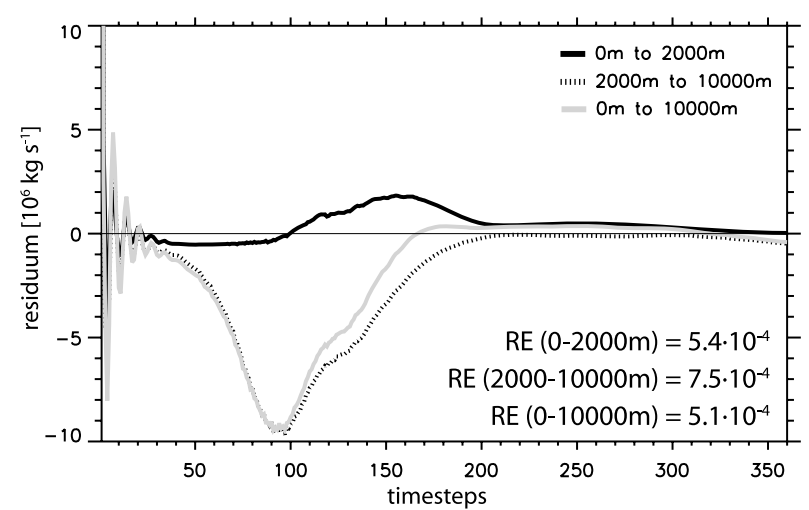

Fig. 8. Case WKRAIN. Residua of the total mass $\left(\mathrm{kg} \mathrm{s}^{-1}\right)$ shown for various locations of vertical extents of the control volume.

budgets of the total mass by dividing the standard $\mathrm{CV}$ into two parts. The first part is located in the lower $2000 \mathrm{~m}$, whereas the second part extends vertically from $2000 \mathrm{~m}$ above the ground up to the upper boundary of the standard CV at $10000 \mathrm{~m}$. The residual curves concerning the standard $\mathrm{CV}$, the lower and the upper part are shown in Fig. 8. In contrary to the mass loss in the middle and upper troposphere, an erroneous mass gain is present in the lower atmosphere. Thus, the negative error inside the cloud is partly compensated by the positive error below the cloud. The error below the cloud implies a problem related to the formulation of microphysical equations which will be discussed in the next Section.

The errors in total mass are correlated with energy errors (Fig. 7b). The total energy change is dominated by changes in the internal energy (eq. 18), whereas the potential and kinetic energy contribute only a small amount to the energy change. The total energy decreases during the first half hour (first 90 time steps). As depicted in Fig. 9, the energy fall is coupled to a strong decrease of latent heat energy due to the condensation of water

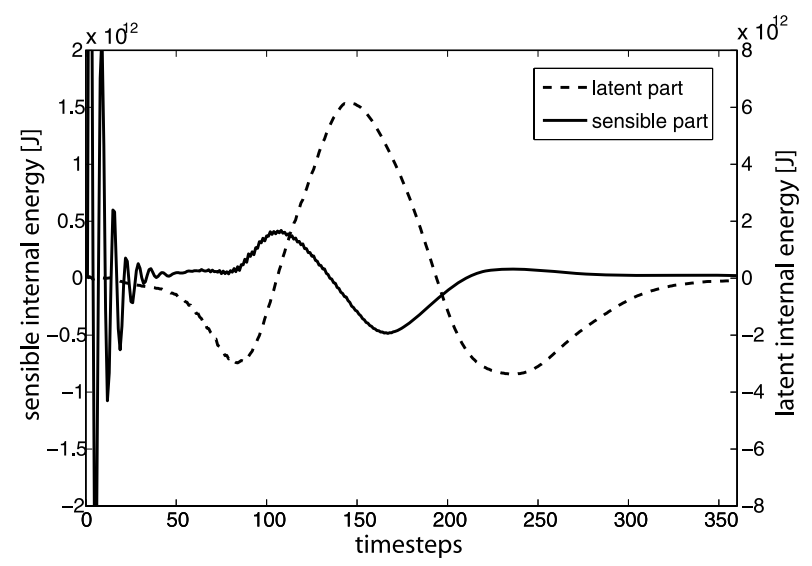

Fig. 9. Case WKRAIN ( $\Delta t=20 \mathrm{~s})$. The change in internal energy $\left(\mathrm{J} \mathrm{s}^{-1}\right)$ integrated over the whole CV. The change is decomposed in the sensible part and latent heat part of internal energy (eq. 18).

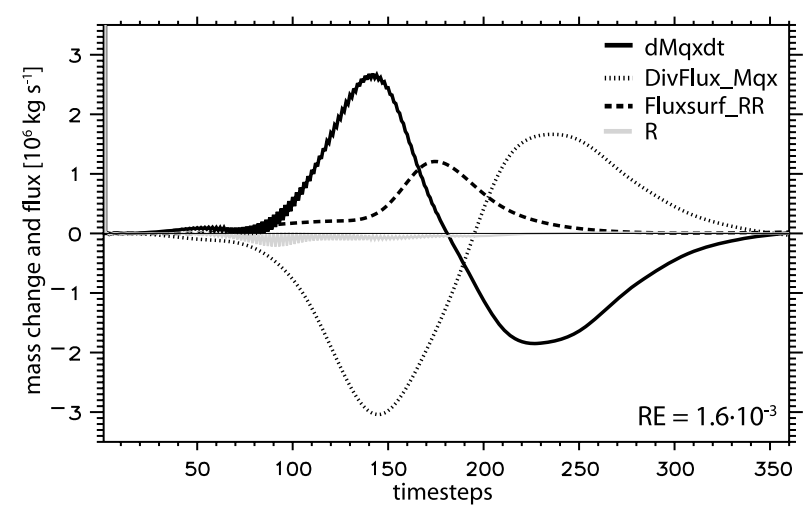

Fig. 10. Case WKRAIN ( $\Delta t=20 \mathrm{~s}$ ). Sub-domain budget of the water mass $\left(\mathrm{kg} \mathrm{s}^{-1}\right)$ concerning the $\mathrm{CV}$. The precipitation flux is denoted by 'Fluxsurf_RR' (eq. 11).

vapour. The latent heat release leads to an increase of sensible heat energy. However, the rise of sensible heat energy is damped by the decrease in total mass and by the initiating evaporation process below and at the top of the cloud. The change of total energy, the energy divergence and the volumic work (third term at the right-hand side of eq. 15) do not cancel each other during the first $90 \mathrm{~min}$.

Starting $30 \mathrm{~min}$ into the forecast, the energy subdomain budget is governed by the change in latent heat energy due to moisture convergence (Fig. 10) and the volumic work of the CV changing the internal energy. Later on, the drying processes along the cold outflow control the change in internal energy. At the end of the simulation the diabatic heat sources are smaller and the residuum of energy is nearly zero.

In contrast to the total mass and energy, the water mass is well conserved (Fig. 10). The moisture divergence, the change in water mass and the precipitation flux are compensating each other. One reason for this good result is that the BOTT advection algorithm is written in a conservative flux form and thus it is preferred to have good conservation skills.

\subsection{Sensitivity of subdomain budgets to moisture advection schemes}

In order to study the performance of the two moisture advection schemes implemented in the COSMO model, we analyse the subdomain budgets concerning the simulation of the case WKRAIN. We test the BOTT scheme and the Semi-Lagrange advection scheme (SL hereafter). The results are shown in Fig. 11 for the SL scheme (thick lines) and the BOTT scheme (thin lines). Applying the SL scheme for moisture transport, the moisture convergence induced by the density current is not as strong as for the BOTT scheme. The maximum values of divergence differ by $10 \%$ after 140 time steps. Nevertheless, the SL scheme produces higher precipitation rates during the first hour. Using the SL scheme we found a reduced generation of cloud water in the mid troposphere which leads to less buoyancy in the up- 


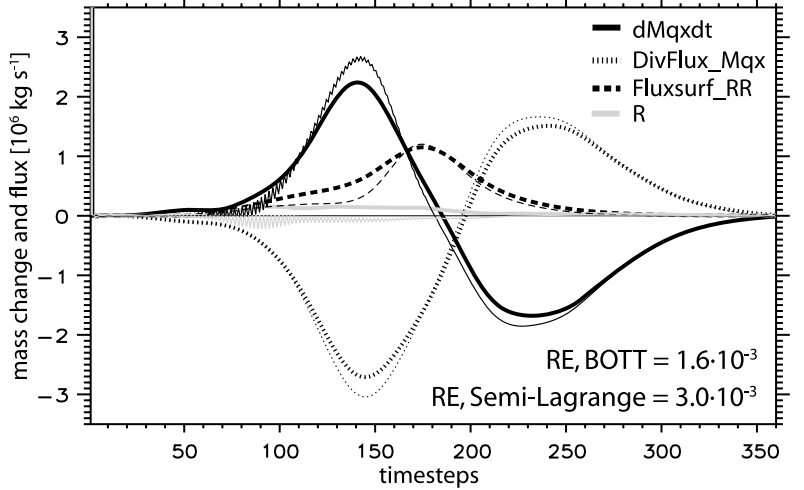

Fig. 11. Case WKRAIN $(\Delta t=20 \mathrm{~s})$. Subdomain budget of the water mass $\left(\mathrm{kg} \mathrm{s}^{-1}\right)$ concerning the $\mathrm{CV}$. Mass change, mass divergence, precipitation flux and residuum using the Semi-Lagrange scheme (thick lines) and the BOTT advection scheme (thin lines). The RE is given in the lower right.

draft channel relative to the BOTT scheme. This implies that the entrainment, respectively the evaporation, at the lower cloud edges is reduced and the sedimentation of rain water is enhanced relative to the BOTT scheme.

It is beneficial for the SL scheme that it does not reveal splitting errors, whereas the RE in water mass conservation is about a factor of two bigger than for the BOTT scheme $\left(3.0 \times 10^{-3}\right.$ versus $1.6 \times 10^{-3}$ ). Of course, the SL scheme is not (water-) mass conserving. Only the global mass over the whole model domain is conserved by a simple multiplicative filling technique (e.g. Staniforth and Cote, 1991). We can speculate that the aforementioned filling technique is not conservative in terms of subdomain water budgets.

\subsection{Sensitivity of subdomain budgets to numerical filters}

In order to test the influence of the numerical filter mechanisms on the subdomain budgets, simulations are carried out in the framework of a modified WKRAIN case. That is, the precipitation physics is switched off and the CAPE is reduced to $350 \mathrm{~J} \mathrm{~kg}^{-1}$ to prevent from strong wind gradients. We run the model with and without Laplacian diffusion ('diff' and 'nodiff') and horizontal divergence damping ('damp' and 'nodamp'). The standard experiment is 'diff,damp'.

Clearly, the boundary fluxes are modified by the horizontal diffusion and also by the divergence damping. However, the budgets themselves are not significantly deteriorated. Simulations without diffusion are affected from instabilities appearing after the strong convection (beginning at time step 220). The sub-domain budget of total mass (Fig. 12), which considers the same CV as in the previous studies, is contaminated. The instability indicates an expected aliasing problem in the model due to missing damping mechanism.

An important result is that the 'nodiff,nodamp' simulation (Fig. 12, straight black line) is much more stable than the 'nod-

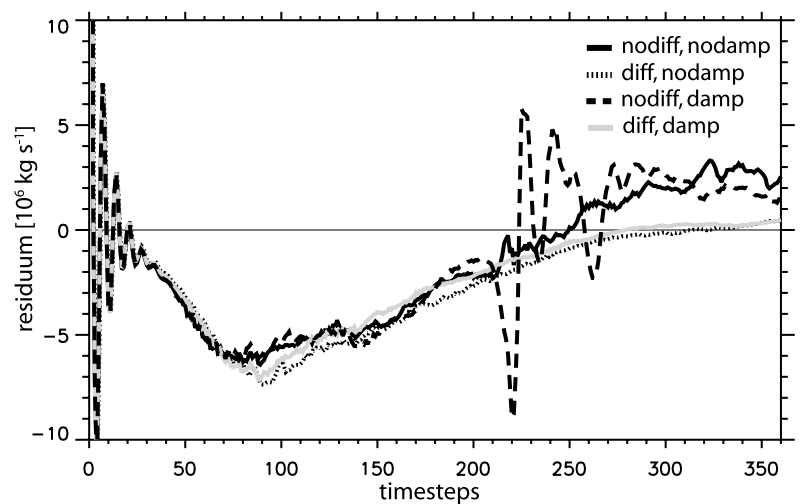

Fig. 12. Case modified WKRAIN ( $\Delta t=20 \mathrm{~s}$ ). The temporal evolution of the residua $\left(\mathrm{kg} \mathrm{s}^{-1}\right)$ of the total mass budget. Results are shown for a run with activated or switched off horizontal diffusion (nodiff, diff) and divergence damping (nodamp, damp).

iff,damp' simulation (Fig. 12, long dashed). After 200 time steps the residual curve of the 'nodiff,damp' simulation is often far away from zero and oscillates. This will increase the integrated residua and therefore worsen the conservation.

As far as we investigated the convective test cases, a specific feature of the SL advection scheme is its sensitivity to the diffusion filter (not shown). There is a significant positive residuum of the water mass conservation, if the diffusive filter is switched off. For example, the RE is $1 \%$ using no filter and $0.3 \%$ using the diffusion filter in the case WKRAIN.

In summary, the diagnostic method used in the cases WKDRY and WKRAIN is very useful and shows limitations of the COSMO model equations for the simulation of resolved convection. To overcome these limitations we modify the model equations and adapt the physical parameterizations to the modified equations. We introduce the model adaptations in the next Section.

\section{A modified saturation adjustment and equation set for the COSMO model: the COSMO-MSA}

In the COSMO model the continuity equation is replaced by a prognostic pressure equation. This does not imply a mass error. However, the law of continuity is only fulfilled, if one uses the complete pressure and temperature equation, e. g. the one of Gassmann and Herzog (2008):

$$
\begin{aligned}
\frac{\partial p^{\prime}}{\partial t}= & -\left(\vec{v} \cdot \nabla_{\zeta}\right) p^{\prime}+g \rho_{0} w-\frac{c_{p}}{c_{v}} p \nabla_{\zeta} \cdot \vec{v} \\
& +\left(\frac{c_{p}}{c_{v}}-1\right) Q_{h}+\frac{c_{p}}{c_{v}} Q_{m},
\end{aligned}
$$

$\frac{\partial T}{\partial t}=-\left(\vec{v} \cdot \nabla_{\zeta}\right) T-\frac{p^{\prime}}{\rho c_{v}} p \nabla_{\zeta} \cdot \vec{v}+\frac{1}{\rho c_{v}}\left(Q_{h}+Q_{m}\right)$, 
instead of the approximated ones applied in the COSMO model (eqs. A1 and A2). The diabatic source term $Q_{h}$ contains the heating rates due to radiation, turbulent sensible heat fluxes and phase changes controlled by cloud microphysics. $Q_{m}$ means the mass redistribution as a result of these diabatic processes:

$$
\begin{aligned}
Q_{m} & =R_{d} T \rho \frac{d \alpha}{d T}, \\
\alpha & =\left(\frac{R_{v}}{R_{d}}-1\right) q_{v}-q_{c}-q_{i}-q_{r}-q_{s}-q_{g}
\end{aligned}
$$

Due to the fact, that the equation sets (A1) and (A2) and (20) and (21) differ from each other, modifications have to be introduced to the COSMO model. A new saturation adjustment technique has to be applied, which for example allows for the adjustment of the pressure to temperature changes due to diabatic heat sources. This process is not considered in the standard saturation adjustment scheme (it refers to eqs. A1 and A2). The new saturation adjustment technique is presented in the following Section. According to the simplified thermodynamics of the COSMO model we will set the specific heat capacities $c_{p}$ and $c_{v}$ to the constant values $c_{p d}$ and $c_{v d}$.

\subsection{A saturation adjustment scheme under fixed volume condition}

The saturation adjustment technique calculates the nucleation rate $S^{c e}\left[\mathrm{~kg} \mathrm{~kg}^{-1} \mathrm{~s}^{-1}\right]$ of cloud water from the vapour phase and vice versa. The released latent heat contributes to the diabatic heat source $Q_{h}$ (eqs. 21 and 20). The parameterization in COSMO is used for non-precipitating cloud types. If mixedphase and ice-phase clouds occur, cloud ice has to be included in the adjustment technique (Lord et al., 1984). Such an extended formulation is not used in the COSMO model. The nucleation and depositional growth of cloud ice are separately formulated in the microphysical schemes (Doms et al., 2002).

The standard saturation adjustment process (SA) is assumed to be isobaric and changes the temperature in an atmosphere at rest following the temperature eq. (A2) (Doms et al., 2002, Section 5) in a consistent way. Applying the standard scheme leads to a residuum in the continuity equation (applying the eqs. A3, A1, A2 and 22 to the continuity equation), that is,

$$
\frac{\rho T R_{v} S^{c e}}{p}-\frac{l_{v} \rho S^{c e}}{T \rho c_{p d}}=-\frac{Q_{m}}{p}-\frac{Q_{h}}{T \rho c_{p d}} .
$$

Using a saturation adjustment technique based on the mass and energy consistent eqs. (20) and (21) we derive a new adjustment for temperature and pressure (the MA approach), which differs from the SA scheme as follows:

\begin{tabular}{l|l} 
SA & MA \\
\hline$\frac{\partial T}{\partial t_{p h y}}=\frac{Q_{h}}{\rho c_{p d}}$ & $\frac{\partial T}{\partial t_{p h y}}=\frac{1}{\rho c_{v d}}\left(Q_{h}+Q_{m}\right)$ \\
$\frac{\partial p}{\partial t_{p h y}}=0$ & $\frac{\partial p}{\partial t_{p h y}}=\frac{c_{p d}}{c_{v d}}\left(Q_{h}+Q_{m}\right)-Q_{h}$,
\end{tabular}

where $Q_{h}=l_{v} \rho d q_{c} / \mathrm{d} t=l_{v} \rho S^{c e}$ denotes the latent heat release. $Q_{m}=-T R_{v} \rho \mathrm{d} q_{c} / \mathrm{d} t=-T R_{v} \rho S^{c e}$ indicates the mass redistribution of water species. The redefined values of cloud water, vapour, temperature and pressure do not change the water content $q^{T}=q_{v}+q_{c}$ and the density. That is, the same mass remains in the same volume, which is a fixed grid box size in the model domain. In comparison to the SA scheme, the internal energy $e$ is also kept constant in the MA scheme:

$\rho \frac{\mathrm{d} e}{\mathrm{~d} t}+p \nabla \cdot \mathbf{v}=\rho c_{p d} \frac{\mathrm{d} T}{\mathrm{~d} t}-\frac{\mathrm{d} p}{\mathrm{~d} t}-l_{v} \rho \frac{\mathrm{d} q_{c}}{\mathrm{~d} t}$.

Thus, cloud water generation increases the sensible heat at the expense of the latent heat $c_{v} \delta T=-l_{v} \delta q_{v}$.

If other prognostic variables than $p$ and $T$ are present, the adjustment principle will differ from (23). Satoh (2003) uses the sensible part of internal energy and density for the forecast and solves equations in terms of internal energy for the adjusted temperature.

The MA approach considers two cases: the air parcels in a grid box are saturated or not. Assuming that all the cloud water is evaporated, the mixture content can be written as $q^{T}=q_{v}^{n}+q_{c}^{n}$ before the adjustment process and $q^{T}=q_{v}^{n+1}=q_{v}^{n}+q_{c}^{n}$ after this process (time index $n$ ). Taking the temporally discretized equation set (23) and using the equation of state, leads to a temperature equation

$$
T^{n+1}=\frac{T^{n}\left(q_{d} R_{d}+q_{v}^{n} R_{v}\right)-c_{p} T^{n}+l_{v} q_{c}^{n}}{\left(q_{d} R_{d}+q_{v}^{n+1} R_{v}\right)-c_{p}} .
$$

If the specific saturation humidity with respect to water vapour $q_{v}^{\text {sat }}(T)=p_{v}^{\text {sat }}(T) /\left(\rho R_{v} T\right)$ at temperature $T^{n+1}$ is higher than $q_{v}^{n+1}$, all water is contained as vapour and the result reads

$$
\begin{aligned}
& q_{v}^{n+1}=q_{v}^{n}+q_{c}^{n}, \quad q_{c}^{n+1}=0, \\
& p^{n+1}=\rho R_{d} T^{n+1}\left\{1+\left(\frac{R_{v}}{R_{d}}-1\right) q_{v}^{n+1}-q_{x}\right\},
\end{aligned}
$$

where $q_{x}$ denotes the remaining water species.

If supersaturation occurs, vapour and cloud water are in phase equilibrium. The value for $q_{v}^{n+1}$ is limited to $q_{v}^{\text {sat }}\left(T^{n+1}\right)$ and the cloud water content can be obtained from the difference $q_{v}^{n}+$ $q_{c}^{n}-q_{v}^{\text {sat }}\left(T^{n+1}\right)$. In this case the derived temperature relation from set (23) is given by

$$
\begin{aligned}
& q_{v}^{\mathrm{sat}}\left(T^{n+1}\right)\left[R_{v} T^{n+1}-l_{v}\right] \\
& \quad+T^{n+1}\left[R_{d}\left(1-q_{v}^{n}-q_{c}^{n}-q_{x}\right)-c_{p}\right]+l_{v}\left[q_{v}^{n}+q_{c}^{n}\right] \\
& \quad=T^{n}\left[q_{d} R_{d}+q_{v}^{n} R_{v}\right]-c_{p} T^{n}+l_{v} q_{c}^{n} .
\end{aligned}
$$


It offers a transcendental equation for temperature, that can be solved with the Newton iteration method. Afterwards, the specific saturation humidity at time step $n+1$ can be calculated with $T^{n+1}$ using the Taylor series expansion.

$$
\begin{aligned}
q_{v}^{\mathrm{sat}}\left(T^{n+1}\right)=q_{v}^{n+1} & =q_{v}^{\mathrm{sat}}\left(T^{n}\right)+\left.\frac{\partial q_{v}^{\mathrm{sat}}}{\partial T}\right|_{n}\left(T^{n+1}-T^{n}\right) \\
\frac{\partial q_{v}^{\mathrm{sat}}}{\partial T} & =-\frac{p_{v}^{\mathrm{sat}}(T)}{\rho R_{v} T^{2}}+\frac{1}{R_{v} T \rho} \frac{\partial p_{v}^{\mathrm{sat}}(T)}{\partial T} .
\end{aligned}
$$

$p_{v}^{\text {sat }}$ is the saturation vapour pressure with respect to a water surface. The relation for $p_{v}^{\text {sat }}$ is given by the Magnus formula or can be derived from the Clausius-Clapeyron equation.

\subsection{Adaption of the physical core of the model}

In order to apply the equations eq. (20) and (21) in COSMO we have to adapt the model formulation to the integration of the $Q_{h}$ and $Q_{m}$ terms. This has to be done not only for the saturation adjustment, but for all physical parameterizations.

The diabatic tendencies computed by the physical parameterizations can be decomposed into cloud microphysics ('cmp'), radiation ('rad') and turbulence ('tur'). The diabatic tendency $Q_{h}$ is

$Q_{h}=Q_{h}^{\mathrm{cmp}}+Q_{h}^{\mathrm{rad}}+Q_{h}^{\mathrm{tur}}$.

The integration procedure in the COSMO model is very elaborated with respect to the temporal coupling between the adiabatic core and the physical parameterizations. We refer to Steppeler et al. (2003), Doms and Schaettler (2002) and Doms et al. (2002).

We modified the tendencies given by eq. (29) in the physical parameterizations in such a way that they coincide with the eqs. (20) and (21). That is, $Q_{h}$ is additionally considered in the pressure equation and $Q_{m}$ is considered as a ten- dency in the pressure and temperature equation. $Q_{m}$ is given by eq. (22).

For simplification we do not consider the term $Q_{h}^{\text {tur }}$ in terms of its influence on the pressure equation. Furthermore, the contribution of $Q_{m}$, which considers turbulent processes, is not taken into account.

The modifications presented in Subsections 5.1 and 5.2 are implemented in a new COSMO model named COSMO-MP.

\section{Subdomain budgets using the modified model COSMO-MP}

\subsection{Case WKRAIN: convection with precipitation}

In order to study the influence of the new model version COSMO-MP introduced in the previous Section on the model simulations the case WKRAIN (see Section 4.4) is again considered. We perform the same subdomain budget analysis as it was done in Section 4.4 using the diagnostic approach from Section 2.

In Fig. 13 the total mass and energy budget is shown. Considering the first half hour of the model run, the COSMO-MP simulates a mass and energy divergence that compensates the occurring decrease of total mass and energy. As already mentioned in Section 4.4, the generation of cloud water is the main process during the first $45 \mathrm{~min}$ (135 time steps). It is controlled by the saturation adjustment. The modified adjustment implemented in COSMO-MP does not adjust only the temperature during phase transitions. It influences additionally the pressure field due to diabatic heating. The resulting pressure gradients between a cloudy and cloud-free grid box lead to an airflow. Therefore, the phase transitions in a grid box modify the fluxes at the boundaries of the grid cell. The MP scheme induces a divergent flow and an expanding volume that is consistent to a condensation, respectively heating, in the centre of the grid box.
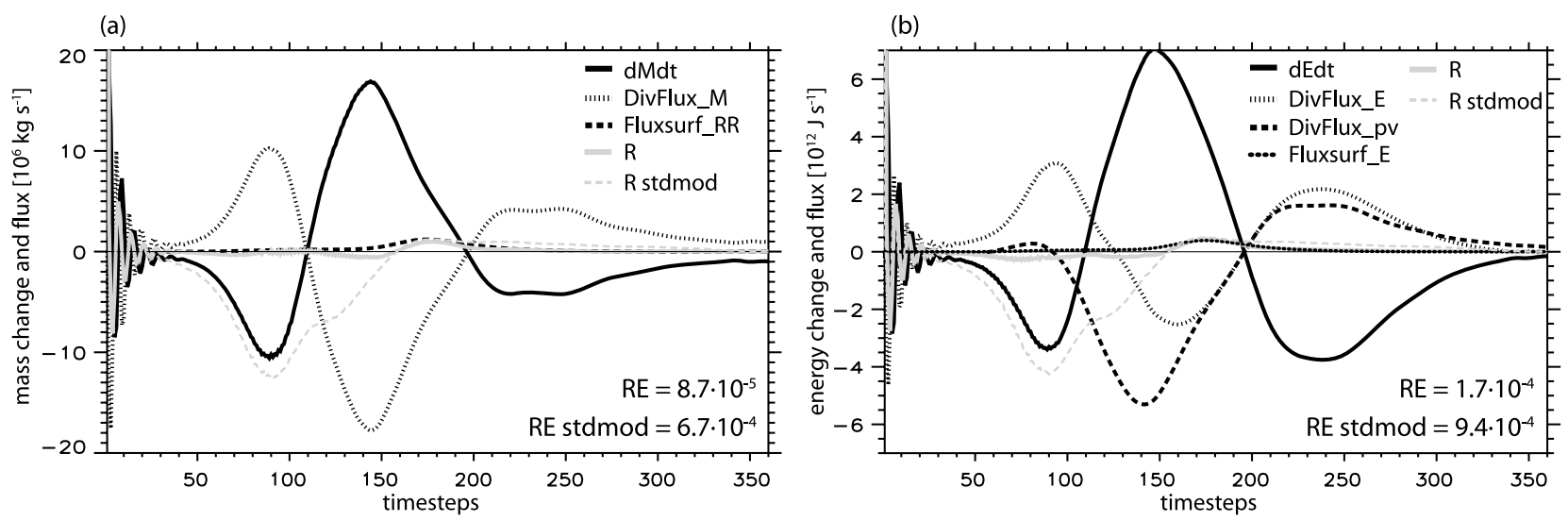

Fig. 13. Case WKRAIN ( $\Delta t=20 \mathrm{~s}$ ). Sub-domain budgets of the total mass and energy concerning the CV. (a) and (b) as in Figs $7 \mathrm{a}$ and $7 \mathrm{~b}$ but using the COSMO-MP model. For comparison, the residual curves and the RE of the standard model are given, named 'R stdmod' and 'RE stdmod', respectively. 
Table 5. RE of energy and mass budget against the CAPE for case WKRAIN

\begin{tabular}{lcclcc}
\hline & \multicolumn{2}{c}{ Mass } & & \multicolumn{2}{c}{ Energy } \\
\cline { 2 - 3 } \cline { 5 - 6 } CAPE & COSMO & COSMO-MP & & COSMO & COSMO-MP \\
\hline $288 \mathrm{~J} \mathrm{~kg}^{-1}$ & $3.8 \cdot 10^{-4}$ & $8.7 \cdot 10^{-5}$ & & $5.2 \cdot 10^{-4}$ & $1.4 \cdot 10^{-4}$ \\
$611 \mathrm{~J} \mathrm{~kg}^{-1}$ & $5.0 \cdot 10^{-4}$ & $8.5 \cdot 10^{-5}$ & & $6.8 \cdot 10^{-4}$ & $1.4 \cdot 10^{-4}$ \\
$1037 \mathrm{~J} \mathrm{~kg}^{-1}$ & $6.7 \cdot 10^{-4}$ & $8.9 \cdot 10^{-5}$ & & $9.3 \cdot 10^{-4}$ & $1.7 \cdot 10^{-4}$ \\
\hline
\end{tabular}

Note: Values listed for standard model and COSMO-MP.

This has a positive impact on the total mass and energy budgets (for comparison see Figs 13 and 7).

Using the diagnostics of subdomain budgets helps to quantify the positive impact of the new physical formulation in the COSMO-MP model. The residuum of total mass and energy is significantly smaller in comparison to the standard COSMO model. The RE is reduced by nearly one magnitude using the COSMO-MP (the numbers in the lower right of the Fig. 13). The improvement factor is increasing, if the amount of CAPE in the atmosphere becomes bigger. In Table 5 the RE is listed in dependence of the CAPE.

Not only the modified saturation adjustment, but also the modified microphysical core of the COSMO-MP model has a positive impact on the simulation. As already mentioned in Section 4.4 , in the course of the simulation the generation of rain water and its evaporation in the lower troposphere will be the dominant physical processes. Using the COSMO-MP instead of the standard model, the cooling in the downdraft due to evaporation of rain water does influence the pressure inside the grid cells of the model. Induced by these pressure adjustments the COSMO-MP simulates airflows in the evaporation regions, but the standard model does not. Consequently, the standard model erroneously gains mass in the lower atmosphere (Fig. 8), whereas the COSMO-MP does not produce any significant error (for comparison see the residual curves named ' $R$ ' and ' $R$ stdmod' in Fig. 13a).

The COSMO-MP improves the conservation properties. The implementations introduced in Section 5 qualifies the COSMO model to simulate physical processes on scales of resolved convection, which could not be handled by the model equations of the standard COSMO. Nevertheless, there is one error which remains in the model. The error is reflected by the imbalance in the total mass and energy budget between time step 150 and 200 (Fig. 13). The assumption of a vanishing vertical velocity normal to the ground results in an artificial mass gain by overestimating the positive pressure change near the surface (see the discussion of Wacker et al., 2006).

The difference between the standard model and the COSMOMP in terms of the precipitation rate is shown in Fig. 14. The COSMO-MP simulates less rain until 50 min compared to the

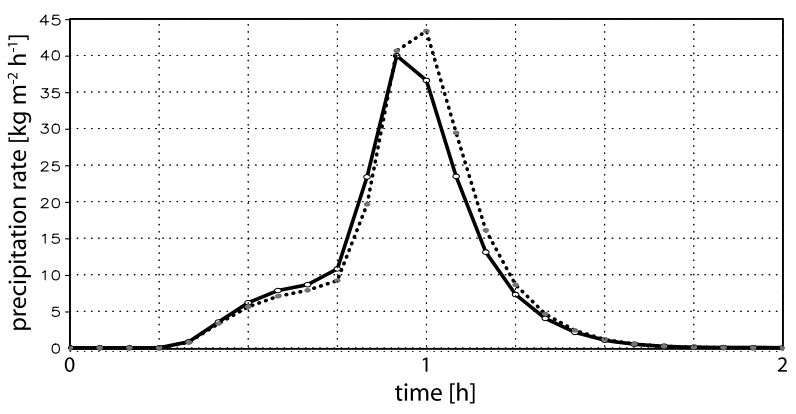

Fig. 14. Case WKRAIN. Precipitation rate $\left(\mathrm{kg} \mathrm{m}^{-2} \mathrm{~h}^{-1}\right)$ as a 16 grid point average using the standard model (solid line) and the COSMO-MP (dashed line).

standard model. Later on, the rain rates are slightly higher for the COSMO-MP. The difference might originate from the modified saturation adjustment of COSMO-MP, which releases more latent heat and increases the maximum vertical velocities relative to the standard adjustment (here not shown). That is, the sedimentating rain water flux in subcloud layers is damped by an enhanced intake of moist air, which drops the precipitation rate relative to the standard model. In the subsequent process, the increased generation of cloud water leads to a stronger precipitation rate using the COSMO-MP. Nevertheless, the RE of the water mass budget does not change significantly using the COSMO-MP model instead of the standard model.

\subsection{Case REAL: realistic test case}

Our budget analysis is applied to a real test case to investigate error magnitudes of an operational forecast. The high resolution COSMO model (COSMO-DE, $\Delta x \simeq 2.8 \mathrm{~km}$ ) is nested into a low resolution model version (COSMO-EU, $\Delta x \simeq 7.6 \mathrm{~km}$ ). A forecast of 30 hours is performed for the 12th August 2002 starting at 0 UTC using the COSMO-MP model with BOTT advection (BOTT-MP) and the standard model with BOTTadvection (BOTT-SM). Additionally, we run the standard model with SL advection (SL-SM).

6.2.1. Synoptic overview. On the investigated day heavy rainfall occurred in the region near the German Erzgebirge. A few days later, a flash flood took place in the Erzgebirge, causing heavy floods along the river Elbe.

The lee cyclone 'ILSE' developed during 11 August 2002 to the south of the Alps and advected moist and warm air, originating from the Mediterranian Sea. 'ILSE' propagated as a so-called Vb cyclone from Italy via the Czech Republic to Poland. At 12 a.m. on 12 August its centre was located in southwest Poland, which is illustrated in Fig. 15a. Its occlusion separated the cold air to the west from the moist and warm air to the east. Due to the long lasting phase of orographic lifting and the almost stationary location of 'ILSE' over southwestern Poland high amounts of 

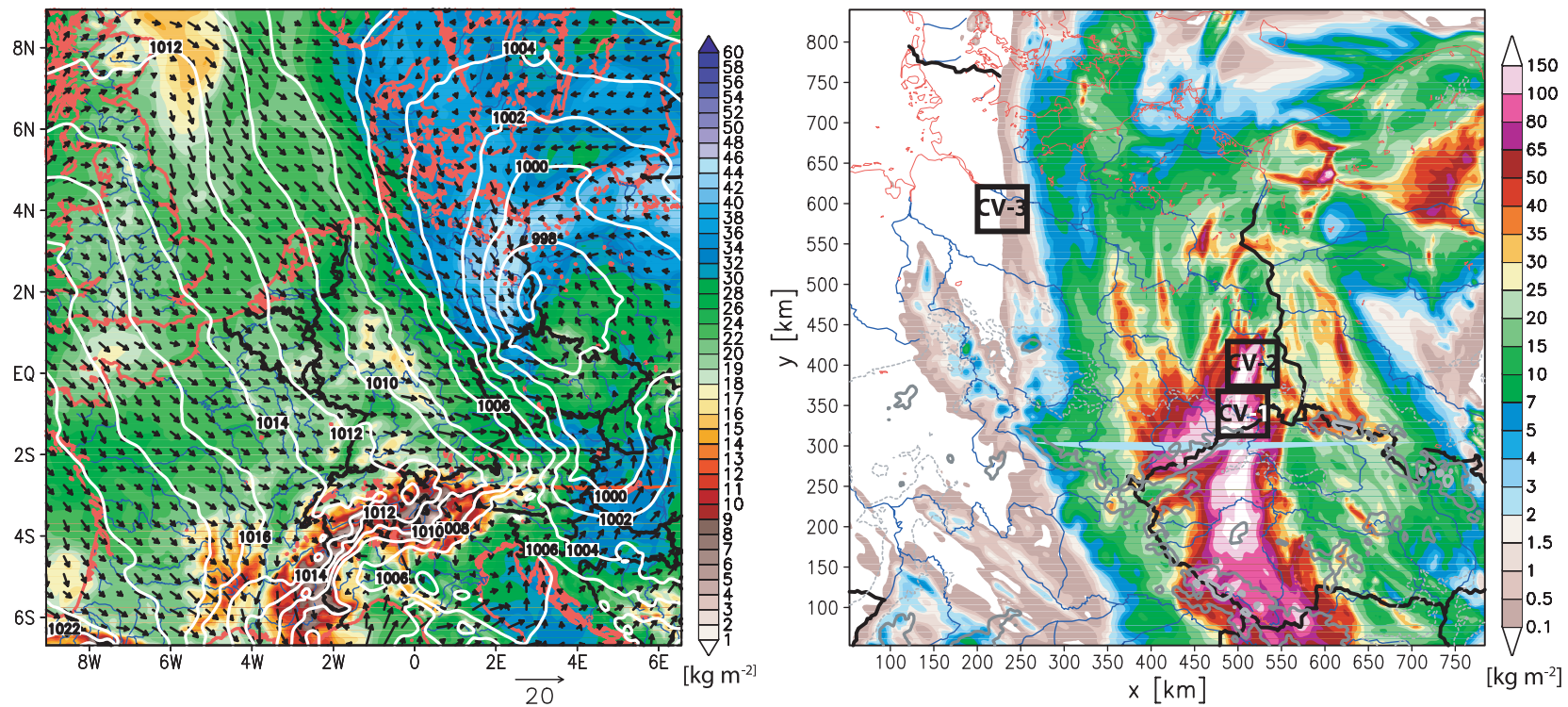

Fig. 15. Real test case of 12 August 2002. (Left panel) COSMO-EU analysis at 12 UTC for the sea level pressure (hPa) (contour), the column-integrated water vapour $\left(\mathrm{kg} \mathrm{m}^{-2}\right)$ (shaded) and the wind vector $\left[\mathrm{m} \mathrm{s}^{-1}\right]$ at $10 \mathrm{~m}$ height. (Right panel) high-resolution COSMO-DE forecast. The 24 hour accumulated precipitation $\left(\mathrm{kg} \mathrm{m}^{-2}\right.$ ) (shaded) from 06 UTC of 12 August 2002 until 06 UTC of 13 August 2002. Additionally, the contour line of $500 \mathrm{~m}$ height is displayed (gray line). The investigated control volumes (CV) are denoted by a black rectangle, numbers are the same as used in the text.

precipitation occurred in the region of Erzgebirge (model result, Fig. 15b). In Zinnwald-Georgenfeld $312 \mathrm{~mm}$ rain was measured within $24 \mathrm{~h}$ (07-07 MEZ).

6.2.2. Subdomain budget analysis. The budget analysis is carried out in different CVs, shown in Fig. 15b. Each CV has a horizontal extension of $30 \mathrm{GP}(80 \mathrm{~km} \times 80 \mathrm{~km})$ and extends from the surface to $9.8 \mathrm{~km}$ height. The functionality of the diagnostic tool for mountainous regions was already tested with an ideal shifting inside the COSMO grid (Baldauf, 2008).

The first $\mathrm{CV}$ covers the region windward to the Erzgebirge with Zinnwald-Georgenfeld in its centre. When the low reaches the Erzgebirge, the moisture flux into CV-1 becomes stronger than the outgoing moisture flux (8 UTC, Fig. 16a). This leads to a 3-D moisture flux convergence, which strengthens until 15 UTC. High amount of precipitation occurs. Afterwards the low moves eastwards in the forecast (slightly faster than it was observed) and the precipitation rates decrease.

During the first hours of slight pre-frontal rain and convection, which is diagnosed by oscillations in the curves of water mass change and water mass convergence, the water mass is well conserved using all three model configurations (BOTT-MP, BOTT-SM and SL-SM). During the strong precipitation event, which is characterized by long phases of high precipitation and convergence rates (from 10 UTC until midnight, Fig. 16a), the performance is decreasing using BOTT-SM and SL-SM. The RE is $23 \%$ using the BOTT-SM model (brown line, Fig. 16a) and $16 \%$ using the SL-SM model (grey line). From the point of view of the subdomain diagnostics, both simulations lost a significant part of the water mass in the CV-1 within 1 day $23 \%$ RE in water mass are equal to $10 \mathrm{~kg} \mathrm{~m}^{-2}$ precipitable water. This might partly explain, why the simulated precipitation differs from the observations in the Zinnwald region. In contrast to the ideal cases, the SL advection performs better than the BOTT advection, which might hint at the pros of an SL scheme in mountainous regions. It produces no splitting errors, as well (straight residual curve, Fig. 16a).

The water mass budget is much better using the BOTT-MP configuration (blue line, Fig. 16a). The water mass loss during the heavy precipitation event is significantly decreased. The RE reduces to $8 \%$. Due to the improvement in water mass conservation we further investigated the precipitation forecast. In Fig. 17 a comparison between observations and model results is shown. The precipitation at Zinnwald-Georgenfeld is increased by $30 \%$ using the BOTT-MP configuration instead of BOTT-SM. This seems to be much more realistic, but we will not draw a conclusion about the forecast skills of both models. Here we only want to mention that the COSMO-MP simulation is physically consistent. Moreover, we test the hypothesis of Dudhia (1993) about the problem of an unrealistic warming of the middle and upper troposphere due to the influence of diabatic effects on the pressure concerning those models that apply a rigid lid condition. However, we could not find a signal indicating the aforementioned problem when comparing the model with radio soundings (here not shown).

The total mass and energy is not well conserved in the simulations (see Figs 16c and 16d), but there is a big improvement in the simulations using the BOTT-MP configuration (compare the brown with the blue lines). Although the RE of total mass is $14 \%$ 

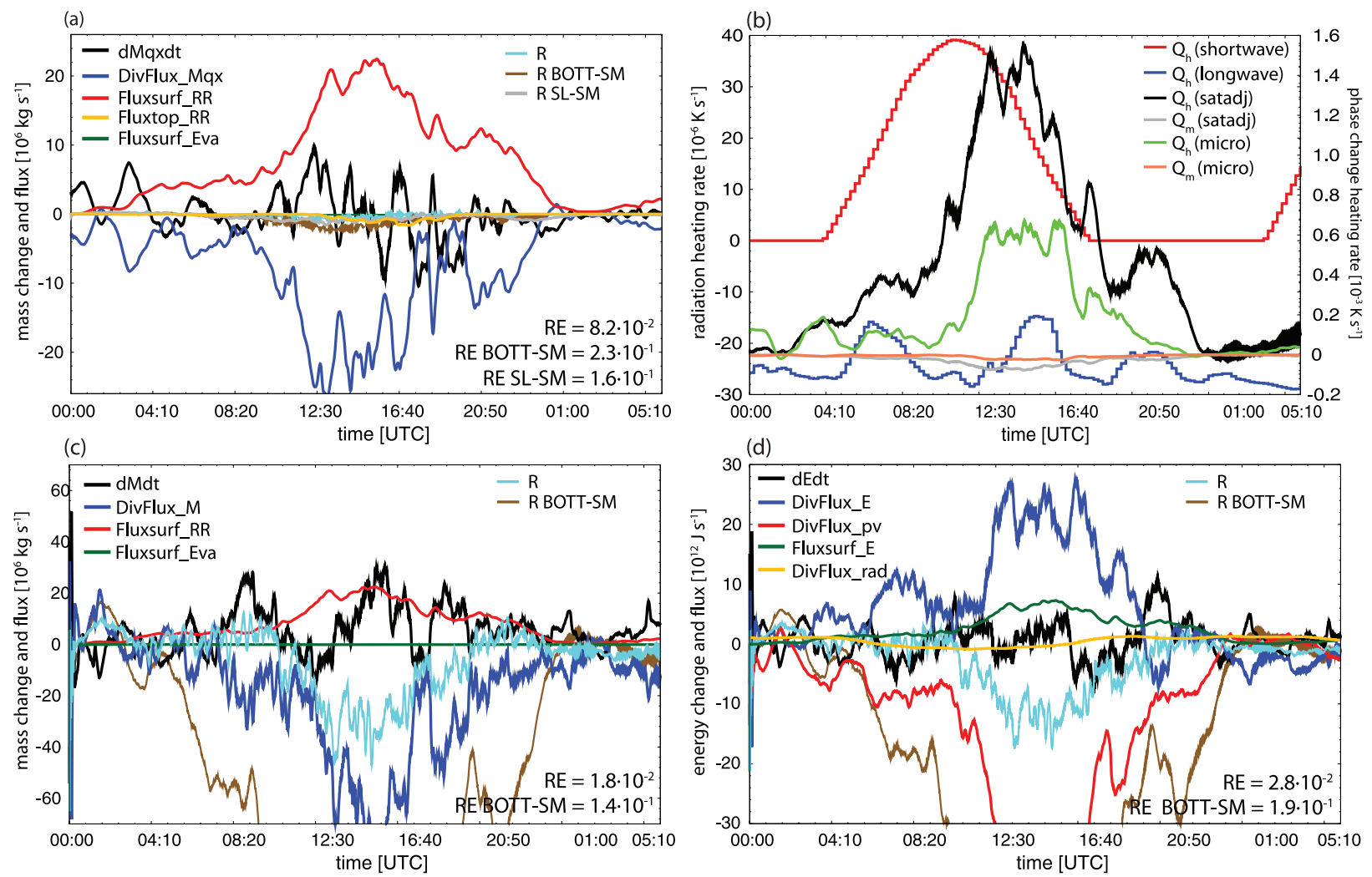

Fig. 16. Real test case of 12 August 2002. Subdomain budgets of the CV-1 in the region Zinnwald-Georgenfeld using the BOTT-MP scheme. The residual curves and the RE of the BOTT-SM and the SL-SM model are denoted by 'w. BOTT-SM' and 'w. SL-SM'. (a) Water mass budget (kg s ${ }^{-1}$ ) involving precipitation flux (Fluxtop_RR) at the top of the CV and evaporation (Fluxsurf_Eva). (b) Diabatic heat sources (K s ${ }^{-1}$ ) of the temperature eq. (21); splitted into parts concerning radiation (short-wave, long-wave), condensation (satadj) and cloud microphysical processes (micro). (c) Total mass budget $\left(\mathrm{kg} \mathrm{s}^{-1}\right)$ involving evaporation (Fluxsurf_Eva). (d) Total energy budget $\left(\mathrm{J} \mathrm{s}^{-1}\right)$ involving the radiation (DivFlux_rad, eq. 16).

and the one of the energy is 19\% using the BOTT-SM configuration, the residual values are much smaller using BOTT-MP. The residua in the BOTT-SM simulation are almost larger than any physical tendency, which means a big error.

Mass budget errors and diabatic heating are strongly related (Fig. 16b). In addition to the latent heat release and latent heat storage from phase changes (black and green line in Fig. 16b), also radiative heating (red line) is relevant in the real simulation. Therefore, errors in mass and energy conservation of the BOTT-SM scheme can also be explained by shortcomings in simulating the expansion and contraction of a CV by short-wave and long-wave radiation. However we cannot investigate these shortcomings, because the radiative forcing is smaller than the cloud microphysical forcing (Fig. 16b) during the whole simulation. We only note, that the COSMO-MP model is able to resolve these radiative processes. Nevertheless, minor errors are still there when using the BOTT-MP. We speculate that the error can be further reduced, if all physical temperature tendencies are analysed for the real case and a solution is found to make the computations in the dynamics and in the physics coherent (according to eqs. 21 and 20).
The transport of turbulent sensible heat fluxes and turbulent momentum fluxes (which influence the kinetic energy) do not significantly contribute to the energy budget (eq. 15). They are at least one dimension smaller than the radiative fluxes.

Beside the conservation analysis, the budget curves point out processes like warm air advection or the formation of snow by an increasing of (sensible internal) energy and the drying of air masses by a decrease of (latent) energy.

We also investigated another region in South Brandenburg (CV-2, here not shown), where high precipitation amounts occurred (Fig. 15). Strong convective rain was formed in the afternoon in the potentially unstable air mass at the occlusion of the low. As already analysed for the CV-1, significant errors in the water mass conservation occur during the period of strong convective rain (BOTT-SM: mass loss of $11 \%$, SL-SM: mass gain of $10 \%)$. The water mass performance is much better applying the BOTT-MP scheme (RE is 5\%).

CV-3 is located to the south of Hamburg. In this region, physical processes are fully different (compare Fig. 18 and 16a). Only slight precipitation is present at the beginning. Due to the impact of a high propagating eastwards a divergent flow is developing 


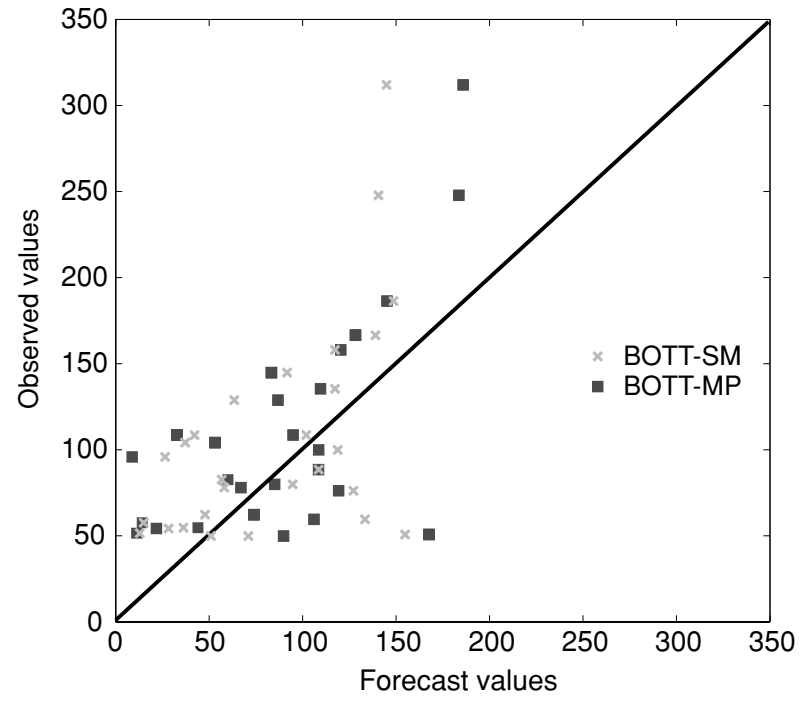

Fig. 17. 24 hour accumulated precipitation from 06 UTC of 12 August 2002 until 06 UTC of 13 August 2002. Scatter plot of the observations and model results using a BOTT-SM (crosses) and a BOTT-MP (rectangles) configuration. Data set of 27 stations near the Erzgebirge.

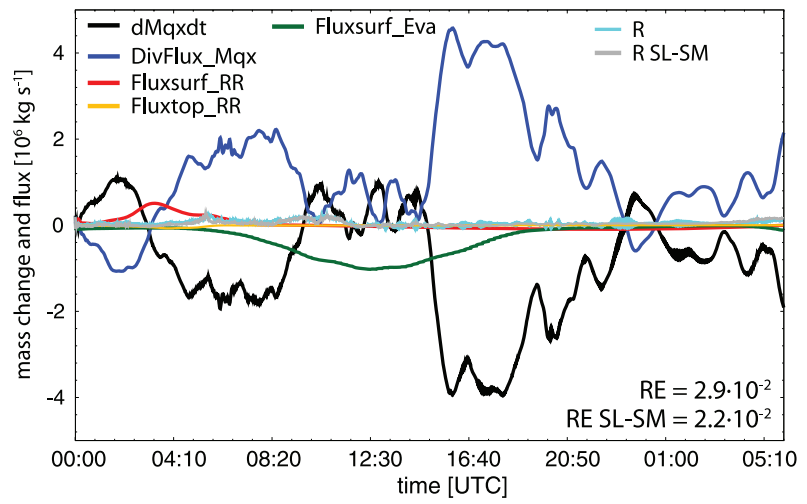

Fig. 18. As in Fig. 16a, but for the region to the south of Hamburg (CV-3). Note the different scaling of the ordinate.

in the morning drying-out the atmosphere, which is depicted in Fig. 18. The clear sky short-wave radiation induces an evaporation flux in the afternoon, that supplies water vapour for the lower atmosphere and leads to small turbulent perturbations reflected by small-scale oscillations in Fig. 18. Mostly all water mass changes and fluxes are balancing each other. The RE is about $2-3 \%$ for all configurations. The differences of the RE are small, because diabatic processes are mostly irrelevant. Only the radiative heating contribute to small errors in total mass and energy, which are less than $1 \%$ for all model configurations.

\section{Conclusion and outlook}

This work demonstrates the construction and application of a 'evaluation approach' by which the subdomain budgets of en- ergy, water mass and total mass can be analysed in a pre-defined $\mathrm{CV}$. As a basic pre-requisite we investigate the diagnostic scheme using different discretization methods in an idealistic test bed. An ideal and numerical shifting of various test functions through a CV has revealed errors originating from discrete grids and flux reconstructions. We defined a configuration for our tool, that minimizes these errors: a Lax-Wendrof or a third-order reconstruction method and a CV size, that exceeds a critical value of $10 \times 10 \times 10 \mathrm{GP}$.

The evaluation method is applied to the mesocale model COSMO. For this purpose we defined the budget equations consistent to the physical formulation of the COSMO. With determining the sources and sinks of mass and energy more complex test cases have been investigated. A rising warm air bubble (Weisman and Klemp, 1982) was used to evaluate the conservation properties of COSMO. The simulations without condensation showed good conservation properties. Slight defects (RE in the order of $10^{-3} \%$ ) occur due to the application of an advective form of the discretized thermo-hydrodynamical equations and due to splitting errors associated with the BOTTtransport scheme.

Taking into account condensation and precipitation processes lead to large errors for the subdomain budgets (RE of $0.1 \%$ within $2 \mathrm{~h}$ ) of total mass and its related quantity energy. These imbalances are associated with the water mass redistribution term and the diabatic term, which contributes to the latent heat release in clouds and to the rain water evaporation in subcloud layers (see also the benchmark of Bryan and Fritsch, 2002). Neglecting these terms in the pressure equation suppresses a balancing flow that is consistent with the heating or cooling in the CV. Therefore, we formulated a new saturation adjustment and we modified the equations concerning microphysical and radiative process. These modifications were implemented in a new COSMO model named COSMO-MP. We improved the mass and energy conservation using the COSMO-MP model. We qualified the model to simulate all relevant physical processes on scales of resolved convection. This includes the correct representation of gravity waves.

Regarding the water mass, we have diagnosed only a slight impact of the large total mass and energy errors for the cases with condensation and rain physics. Accordingly, the improvement of the water mass conservation using the COSMO-MP model instead of the standard model has been less effective (factor 2 ) in comparison to the total mass. The investigation of the real test case showed an improvement factor of 3 , which reduces the error from $10 \mathrm{~kg} \mathrm{~m}^{2}$ to $3 \mathrm{~kg} \mathrm{~m}^{2}$ precipitable water within 24 hours. Additionally, the BOTT scheme has performed better for the Weisman-Klemp case than the SL scheme. The SL advection scheme works particularly worse in case of switched off horizontal diffusion (tends to a large positive bias of 1\%), but seems to be advantageous in mountainous regions.

In order to study the impact of non-physical damping schemes on the subdomain budgets, we have carried out a sensitivity 
analysis concerning the frequently used horizontal diffusion and divergence damping. These numerical techniques that stabilize the model run, have not such a large impact as the physical errors. An exception is the horizontal damping used without diffusion. This configuration seems to be at the limit of stability and leads to large REs.

Some aspects of our results confirm earlier studies, in which the mass deficit due to neglecting some diabatic heat terms in the equations was mentioned (e.g. Doms and Schaettler, 2002; Bryan and Fritsch, 2002). We have shown that the sub-domain analysis of model conservation properties can reveal insight into the model qualities. With the 'evaluation tool' we provide the possibility for diagnosing budgets in any sub-domain inside the model domain. Thus, one can analyse realistic tet cases. We have pointed out that the numerical schemes can impact the conservation properties up to the order of $1 \%$. Furthermore, diabatic processes were identified to be relevant for a reliable mass and energy conservation in a numerical model. The physical treatment of the diabatic processes can impact the conservation properties up to the order of $10 \%$. Since the diabatic processes are quite generic and the related parameterizations are similar in the COSMO and other NWP models, it is relevant to perform similar evaluations for other models. This statement also holds for those written in flux form.

Concerning the operational COSMO model, an alternative formulation of the equations and physical parameterizations as presented in this paper, might have an positive impact on the model results. A saturation adjustment approach based on the method presented here is tested and evaluated at the German Weather Service in the framework of a pre-operational version. Indeed, the preliminary studies indicate that the quality of the forecast is improved compared to the standard operational model (Baldauf and Blahak, personal communication).

\section{Acknowledgments}

The authors thank Hans-Joachim Herzog (German Weather Service) for his useful comments and for his critical reading of the manuscript. We are grateful to Marco Restelli and Florian Rauser (Max Planck Institute for Meteorology) for the interesting discussions concerning mass and energy conservation and the diagnostic method. We are indebted to Janek Zimmer (University of Leipzig) for providing information on the Elbe flash flood case. We thank the reviewers for their helpful remarks that improve the paper significantly. Last but not least, we thank the DFG (German Research Foundation) for founding this work.

\section{Appendix: Formulation of the COSMO model}

\subsection{Model description}

The COSMO model (Doms and Schaettler, 2002; Doms et al., 2002) is a compressible non-hydrostatic NWP model used for operational forecasts on limited domains by several European weather services. The model solves the non-hydrostatic primitive equation system that approximates a shallow atmosphere [following the Penn State-NCAR Mesoscale Model (MM5, Dudhia, 1993)]. The prognostic variables are the pressure deviation $p^{\prime}$ from the reference state, the temperature $T$, the threedimensional wind field $\mathbf{v}$ and the partial masses of the water constituents $q_{x}$. The dynamic equations of the COSMO model are given in Steppeler et al. (2003, eqs. 9-15). The approximations used to derive the model equations are given in Doms and Schaettler (2002). In the context of the paper we only want to present the temperature and pressure equation:

$$
\begin{aligned}
& \frac{\partial p^{\prime}}{\partial t}=-\left(\vec{v} \cdot \nabla_{\zeta}\right) p^{\prime}+g \rho_{0} w-\frac{c_{p d}}{c_{v d}} p \nabla_{\zeta} \cdot \vec{v}, \\
& \frac{\partial T}{\partial t}=-\left(\vec{v} \cdot \nabla_{\zeta}\right) T-\frac{1}{\rho c_{v d}}\left(p \nabla_{\zeta} \cdot \vec{v}\right)+\frac{Q_{h}}{\rho c_{p d}} .
\end{aligned}
$$

$\nabla_{\zeta}$ denotes the three-dimensional Nabla operator with respect to terrain-following coordinates. The specific heat capacities of moist air at constant pressure $c_{p d}$ and volume $c_{v d}$ are approximated to be valid for dry air. $Q_{h}$ summarizes the diabatic heat sources originating from turbulent heat fluxes, radiation and cloud mircophysics. The density $\rho$ is derived from the equation of state

$$
\begin{aligned}
p & =\rho\left(R_{d} q_{d}+R_{v} q_{v}\right) T \\
& =\rho R_{d}\left\{1+\left(\frac{R_{v}}{R_{d}}-1\right) q_{v}-q_{l}-q_{f}\right\} T,
\end{aligned}
$$

where $R_{d}$ and $R_{v}$ denote the gas constants for dry air and water vapour, respectively. The indices $v, l$ and $f$ stand for the water vapour, the liquid water and the solid water phase.

An overview about the model grid and the numerical discretization is given in Steppeler et al. (2003). We have to note, that the recent dynamical core of the model is discretized using a third-order Runge-Kutta scheme (Wicker and Skamarock, 2002) for time integration, which is combined with a time-splitting method to separate between slow processes like advection and fast processes related to fast wave propagation (as in Wicker and Skamarock, 2002). By default, the horizontal part of the advection operator in the model equations (Steppeler et al., 2003, eqs. 9-15) is discretized with fifth-order upwind scheme. The vertical advection is treated implicitly using a Crank-Nicolson scheme.

The recent cloud microphysical scheme considers six water components: water vapour, cloud water, cloud ice, rain, snow and 'graupel'. The cloud microphysics are represented by a bulk water-continuity model (Doms et al., 2002, Section 5). The precipitation is treated prognostically in the COSMO model. This allows for drifting the hydrometeors during their way to the ground (Gassmann, 2002).

For simulations with grid spacing more than $5 \mathrm{~km}$ the deep and shallow moist convection is parameterized by the Tiedtke 
mass flux scheme (Tiedtke, 1989). For model applications on the meso- $\gamma$ scale with grid spacings less than $5 \mathrm{~km}$ the deep moist convection is explicitly resolved.

The turbulent fluxes are subgrid-scale processes and have to be parameterized. A diagnostic level 2 closure (according to Mellor/Yamada) and two different second-order closure schemes (level 2.5), which apply the prognostic equation of the TKE, are implemented in the COSMO. A more detailed overview on the model physics is given in (Steppeler et al., 2003, section 4) and an extensive description can be found in Doms et al. (2002).

\section{References}

Baldauf, M. 2008. A tool for testing conservation properties in the COSMO-model, COSMO Newsletter No. 7, Deutscher Wetterdienst, Offenbach a. M., Germany.

Bott, A. 1989. A positive definite advection scheme obtained by nonlinear renormalization of the advective fluxes. Mon. Wea. Rev. 117, 1006-1015.

Bryan, G. H. and Fritsch, J. M. 2002. A benchmark simulation for moist nonhydrostatic numerical models. Mon. Wea. Rev. 130, 2917-2928.

Catry, B., Geleyn, J.-F., Tudor, M., Bénard, P. and Trojákova, A. 2007. Flux-conservative thermodynamic equations in a mass-weighted framework. Tellus 59A, 71-79.

Doms, G. 2004. Test of the moisture mass conservation in LM, COSMO Newsletter No. 3, Deutscher Wetterdienst, Offenbach a. M., Germany.

Doms, G. and Schaettler, U. 2002. A description of the nonhydrostatic Regional Model LM - part I: dynamics and numerics, DWD Documentation, Deutscher Wetterdienst, Offenbach a. M., Germany.

Doms, G., Foerstner, J., Heise, E., Herzog, H.-J., Raschendorfer, M. and co-authors. 2002. A description of the nonhydrostatic Regional Model LM - part II: physical parametrization, DWD Documentation, Deutscher Wetterdienst, Offenbach a. M., Germany.

Doswell, C. A. and Rasmussen, E. N. 1994. The effect of neglecting the virtual temperature correction on cape calculation. Wea. Forecast. 9 , 625-629.

Dudhia, J. 1993. A nonhydrostatic version of the penn state-ncar mesoscale model: validation tests and simulation of an atlantic cyclone and cold front. Mon. Wea. Rev. 121, 1493-1513.

Durran, D. R. 1999. Numerical methods for wave equations in geophysical fluid dynamics. In: Texts in Applied Mathematics, 1st Edition. (ed. Durran, D. R.). Springer-Verlag, New York, USA.

Emanuel, K. A. (ed.) 1994. Atmospheric convection, 1st Edition. Oxford University Press, New York.

Ferziger, J. H. and Peric, M. 2002. Computational methods for fluid dynamics. In: Motion and Motion Systems, 3rd Edition (eds. Ferziger, J. H. and Peric, M.). Springer-Verlag, Berlin, Heidelberg.
Förstner, J., Baldauf, M. and Seifert, A. 2006. Courant number independent advection of the moisture quantities for the LMK, COSMO Newsletter No. 6, Deutscher Wetterdienst, Offenbach a. M., Germany.

Gassmann, A. 2002. Nummerische Verfahren in der nichthydrostatischen Modellierung und ihr Einfluss auf die Güte der Niederschlagsvorhersage. PhD thesis, University of Bonn.

Gassmann, A. and Herzog, H.-J. 2008. Towards a consistent numerical compressible nonhydrostatic model using generalized hamiltonian tools. Quart. J. R. Meteor. Soc. 134, 1597-1613.

Lord, S., Willoughby, H. and Piotrowicz, J. 1984. Role of parameterized ice-phase microphysics in an axisymmetric nonhydrostatic tropical cyclone model. J. Atmos. Sci. 41, 2836-2848.

Satoh, M. 2003. Conservative scheme for a compressible nonhydrostatic model with moist processes. Mon. Wea. Rev. 131, 1033-1049.

Schlünzen, K. H. 1997. On the validation of high-resolution atmospheric mesoscale models. J. Wind Eng. Ind. Aerodyn. 67,68, 479-492.

Schlünzen, K. H., Stahlschmidt, T., Rebers, A., Niemeier, U., Kriews, M. and co-authors. 1997. Concept and realization of the mesoscale transport- and fluid-model 'METRAS', METRAS Techn. Rep, Meteorologisches Institut, Universität Hamburg, Hamburg, Germany.

Skamarock, W. C., Klemp, J. B., Dudhia, J., Gill, D. O., Barker, D. M. and co-authors. 2005. A description of the Advanced Research WRF version 2, NCAR Tech. Note, Mesoscale and Microscale Meteorology Devision NCAR, Boulder, Colorado, USA.

Staniforth, A. and Cote, J. 1991. Semi-lagrangian integration schemes for atmospheric model: a review. J. Geophys. Res. 119, 22062223.

Steppeler, J., Doms, G., Schättler, U., Bitzer, H. W., Gassmann, A., and co-authors. 2003. Meso-gamma scale forecasts using the nonhydrostatic model LM. Meteor. Atmos. Phys. 82, 75-96.

Tiedtke, M. 1989. A comprehensive mass flux scheme for cumulus parameterization in large scale models. Mon. Wea. Rev. 117, 17791799.

Wacker, U., Frisius, T. and Herbert, F. 2006. Evaporation and precipitation surface effects in local mass continuity laws of moist air. J. Atmos. Sci. 63, 2642-2652.

Wacker, U. and Herbert, F. 2003. Continuity equations as expressions for local balances of masses in cloudy air. Tellus 55, 247-254.

Weisman, M. L. and Klemp, J. B. 1982. The dependence of numerically simulated convective storms on vertical wind shear and buoyancy. Mon. Wea. Rev. 110, 504-520.

Wicker, L. J. and Skamarock, W. C. 2002. Time-splitting methods for elastic models using forward time schemes. Mon. Wea. Rev. 130, 2088-2097.

Xue, M., Droegemeier, K. K. and Wong, V. 2000. ARPS: a multiscale nonhydrostatic atmospheric simulation and prediction tool. part I : model dynamics and verification. Meteor. Atmos. Physics. 75, $161-193$. 